Draft Version June 4, 2018

Preprint typeset using $\mathrm{L}^{A} \mathrm{~T}_{\mathrm{E}} \mathrm{X}$ style emulateapj v. 08/22/09

\title{
PROBING THE BALANCE OF AGN AND STAR-FORMING ACTIVITY IN THE LOCAL UNIVERSE WITH CHAMP
}

\author{
Anca Constantin $^{1}$, Paul Green ${ }^{1}$, Tom Aldcroft ${ }^{1}$, Dong-Woo Kim ${ }^{1}$, Daryl Haggard ${ }^{2}$, Wayne Barkhouse $^{3}$, Scott \\ F. ANDERSON ${ }^{2}$ \\ Draft version June 4, 2018
}

\begin{abstract}
The combination of the SDSS and the Chandra Multiwavelength Project (ChaMP) currently offers the largest and most homogeneously selected sample of nearby galaxies for investigating the relation between X-ray nuclear emission, nebular line-emission, black hole masses, and properties of the associated stellar populations. We provide X-ray spectral fits and valid uncertainties for all the galaxies with counts ranging from 2 to 1325 (mean 76, median 19). We present here novel constraints that both X-ray luminosity $L_{X}$ and X-ray spectral energy distribution bring to the galaxy evolutionary sequence $H I I \rightarrow$ Seyfert/Transition Object $\rightarrow$ LINER $\rightarrow$ Passive suggested by optical data. In particular, we show that both $L_{X}$ and $\Gamma$, the slope of the power-law that best fits the $0.5-8 \mathrm{keV}$ spectra, are consistent with a clear decline in the accretion power along the sequence, corresponding to a softening of their spectra. This implies that, at $z \approx 0$, or at low luminosity AGN levels, there is an anti-correlation between $\Gamma$ and $L / L_{\text {edd }}$, opposite to the trend exhibited by high $z$ AGN (quasars). The turning point in the $\Gamma-L / L_{\text {edd }}$ LLAGN + quasars relation occurs near $\Gamma \approx 1.5$ and $L / L_{\text {edd }} \approx 0.01$. Interestingly, this is identical to what stellar mass X-ray binaries exhibit, indicating that we have probably found the first empirical evidence for an intrinsic switch in the accretion mode, from advection-dominated flows to standard (disk/corona) accretion modes in supermassive black hole accretors, similar to what has been seen and proposed to happen in stellar mass black hole systems. The anti-correlation we find between $\Gamma$ and $L / L_{\text {edd }}$ may instead indicate that stronger accretion correlates with greater absorption. Therefore the trend for softer spectra toward more luminous, high redshift, and strongly accreting $\left(L / L_{e d d} \gtrsim 0.01\right)$ AGN/quasars could simply be the result of strong selection biases reflected in the dearth of type 2 quasar detections.
\end{abstract}

Subject headings: galaxies: active - galaxies: nuclei - galaxies: emission lines - X-rays: galaxies surveys

1. INTRODUCTION: BH ACCRETION, THE LOW LUMINOSITY AGN, AND X-RAY EMISSION

Understanding the nuclear activity in nearby galaxies is essential for constraining the galaxy formation and evolution process. While energetically unimpressive, the nearby galactic nuclei offer the best case scenario for (1) the most common state of accretion in the current universe, (2) the end-point of quasar evolution, or simply (3) their scaled-down version. Observationally, the low redshift $z \approx 0$ accretion systems are the best testbeds for identifying the processes involved in triggering and further fuelling accretion onto the central black hole $(\mathrm{BH})$ because they offer unique joint investigations of both the nuclear accretion and the properties of the host, i.e., the star-formation (SF).

Unlike the optically luminous quasars, which are radiating close to their Eddington limit (Kollmeier et al. 2006), and only for short $\left(\sim 10^{7}\right.$ yr $)$ times (Yu \& Tremaine 2002), accretion activity in nearby galaxy centers appears extremely diverse, spanning > 6 orders of magnitude in the Eddington ratio, and (maybe consequently) a wide range in their duty-

\footnotetext{
${ }^{1}$ Harvard-Smithsonian Center for Astrophysics, Cambridge, MA 02138

2 Department of Astronomy, University of Washington, Seattle, WA, USA

${ }^{3}$ Department of Physics and Astrophysics, University of North Dakota, Grand Forks, ND 58202, USA
}

cycles (Heckman et al. 2004; Ho 2008). This variety provides empirical constraints to model predictions linking the $\mathrm{BH}$ growth rate and the host bulge formation. The two are indeed connected: it is the younger galaxies that host the more rapidly growing BHs (Heckman et al. 2004; Cid Fernandes et al. 2005; Constantin. Hoyle, \& Vogeley 2008). The exact physical mechanism responsible for this link, and in general for the close interplay between SF and $\mathrm{BH}$ accretion remains however elusive. Simulations place important constraints on different models for the way black holes are fueled, and provide a quantitative and physical distinction between local, low luminosity, weak (or quiescent) AGN activity, and violent, mergerdriven bright quasars (e.g.. Hopkins \& Hernquist 2006, 2009). Environmental studies of the nearby active galactic nuclei are consistent with these ideas of nonmerger-driven fueling for the weak $\mathrm{BH}$ growth observed in the nearby universe (Constantin \& Vogeley 2006; Constantin. Hoyle, \& Vogeley 2008). More recent analysis of the observed distribution of Eddington ratios as a function of the $\mathrm{BH}$ masses provide additional constraints, suggesting that even at $z \approx 0$ there might be two distinct regimes of $\mathrm{BH}$ growth, which are determined by the supply of cold gas in the host bulge. The $\mathrm{BH}$ regulates its own growth at a rate that is independent of the interstellar medium's characteristics as long as the gas is plentiful, but when the gas runs out the BH's growth will be reg- 
ulated by the rate at which evolved stars lose their mass (Kauffmann \& Heckman 2009). These different fueling modes at low luminosities must manifest differently at wavelengths outside the optical regime, allowing further means to constrain and discriminate among them.

The most ubiquitous type of activity at $z \approx 0$ that resembles that of quasars is identified at optical wavelengths as either a narrow-lined Low Ionization Nuclear Emission Regions(LINER, L) or a "transition" (T) object, whose properties border on the definition of a starburst galaxy and an AGN. Emission-line ratio diagnostics (e.g., Baldwin. Phillips, \& Terlevich 1981; Veilleux \& Osterbrock 1987; Kewlev et al. 2006) that have been quite successful in identifying cases where the dominant ionization mechanism is either accretion onto a black hole (i.e., Seyferts, Ss) or radiation from hot, young stars (i.e., H II nuclei), remain inconclusive for the majority of Ls and Ts. The Ls that exhibit quasar-like broad lines (L1s, by analogy with Seyfert 1s) are unambiguously accretion-powered sources, however those lacking these features (the L2s) could have lines generated by shocks, post-starbursts, or other processes unrelated with accretion. Deciphering the underlying emission source(s) of these ambiguous nuclei is an ongoing struggle.

Recent analyses of the emission properties of the low luminosity AGN (LLAGN) in relation to a wide variety of characteristics of their hosts, together with considerations of their small and large scale environments, reveal a sequence $H I I \rightarrow$ Seyfert/Transition Object $\rightarrow$ LINER $\rightarrow$ Passive $(H I I \rightarrow S / T \rightarrow L \rightarrow P$ ) that these objects obey, at least in a statistical sense (Constantin \& Vogeley 2006; Schawinski et al. 2007; Constantin et al. 2008). This sequence traces trends in (1) increasing host halo mass, (2) increasing environmental density, (3) increasing central $\mathrm{BH}$ mass and host stellar mass, (4) decreasing BH accretion rate, (5) aging of the stellar population associated with their nuclei, and (6) decreasing in the amount of dust obscuration, which might translate into a decrease in the amount of material available for star-forming or accretion. This sequence therefore suggests a process of transformation of galaxies from SF via AGN to quiescence, which may be the first empirical evidence for an analogous duty cycle to that of the high $z$ bright systems (i.e., quasars). State of the art hydrodynamical models provide clear support for such a scenario, by showing that during mergers, the $\mathrm{BH}$ accretion peaks considerably after the merger started, and after the star-formation rate has peaked (e.g., Di Matteo et al. 2005, Hopkins et al. 2006). Constraining the nature of this $H I I \rightarrow S / T \rightarrow L$ sequence at $z \approx 0$ will improve our understanding of the degree to which the LLAGN phenomenon fits into the galactic $\mathrm{BH}$ accretion paradigm.

The X-ray emission is, arguably, the most sensitive test for accretion and its intensity and efficiency, and thus, it is of great interest to test and validate this sequence against large homogeneous X-ray selected samples. The Chandra Multiwavelength Project (ChaMP; Green et al. 2004) provides a unique opportunity for this, by providing the largest-area Chandra survey to date, which, when cross-matched with the SDSS, provides an unprecedented number of galaxies in the local Universe for which we can combine and contrast measurements of the X-ray and optical emission. The sample of $\sim 110$ Chandra X-ray detected nearby galaxies (excluding broad line objects) analysed in this study represents a significant improvement in both sample size and homogeneity both for X-ray selection and optical spectral type coverage.

Previous studies of the relation between the X-ray nuclear emission, optical emission line activity and black hole masses provide important physical constraints to the LLAGN phenomenon. Almost invariably, the conclusions are that LLAGN are probably scaled down versions of more luminous AGN (e.g., Ho et al. 2001; Panessa et al. 2006), and that $M_{\mathrm{BH}}$ is not the main driver of the (soft) X-ray properties (Greene \& Ho 2007). The LLAGN are claimed to be X-ray detected at relatively high rates, and are found to be relatively unabsorbed, obscuration appearing to play only a minor role in their detection rates and/or in classifying them as types 1 and 2 in X-rays (Roberts \& Warwick 2000; Halderson et al. 2001; Miniutti et al. 2008; Ho 2008), with the exception of those known to be Compton thick. Nonetheless, the X-ray investigations of AGN activity at its lowest levels remain largely restricted to Ls and Ss.

Deciphering the ambiguous nature of Ls in particular, has been the target of many X-ray studies of LLAGN focused on these sources (Yaqoob et al. 1995; Ishisaki et al. 1996; Ivomoto et al. 1998, 2001; Terashima et al. 1998, 2000a,b, 2002; Pellegrini et al. 2000a ,b, 2002; Georgantopoulos et al. 2002; Ptak et al. 1996, 1999, 2004; Roberts, Schurch \& Warwick 2000). A hard, power-law AGN signal is generally spectrally resolved for the majority of them, however, the corresponding energy (photon) index is marginally steeper (softer) than in (broad line) Ss; many of them require a soft thermal component, that somehow differs from the blackbody soft excess commonly seen in $\mathrm{Ss}$ and quasars. The $\mathrm{Fe} \mathrm{K} \alpha$ emission or the Compton reflection component are usually weak in these sources, indicating that X-ray reprocessing is not by material in an optically thick accretion disk (Lightman \& White 1988; George \& Fabian 1991). Because most of these studies are based on large-beam observations, mostly $A S C A$ or BeppoSAX, Ls' emission has also quite often been attributed to stellar processes. Higher spatial resolution Chandra and XMM-Newton observations (Bohringer et al. 2001; Kim \& Fabbiano 2003; Pellegrini et al. 2003; Terashima \& Wilson 2003; Filho et al. 2004; Page et al. 2004; Starling et al. 2005; Flohic et al. 2006; Gonzalez-Martin et al. 2006; Soria et al. 2006) remain torn between these findings, as the stellar interpretation persists for quite a number of these sources.

In this work we approach the LLAGN phenomenon via the $H \quad I I \rightarrow S / T \rightarrow L \rightarrow$ Passive galaxy evolutionary sequence described above. In particular, we test the validity of the sequence within X-ray selected LLAGN via a large variety of optical emission properties, which first provided evidence for the sequence, and also in their $\mathrm{X}$-ray properties. We combine the ChaMP X-ray detections with a sample of SDSS DR4 nearby galaxies that excludes broad line objects, creating a large sample of galaxy nuclei that spans a range of spectral types, from passive to actively line emitting systems, including the star-forming and accreting types, along with those of mixed or ambiguous ionization. Through measurements 
of their X-ray spectra ${ }^{4}$, fluxes and luminosities, we characterize the sequence in terms of strength and mode of accretion. We provide here the first investigation of the relation between $\Gamma$ and the Eddington ratio $L / L_{\text {edd }}$ at the lowest levels of accretion. We reveal a rather surprising anti-correlation between these two measures, opposite to what luminous AGN and quasars exhibit. This finding reveals a turning point in the general $\Gamma-L / L_{\text {edd }}$ relation followed by AGN, which is identical (within the errors) to that shown by the $\Gamma-L / L_{\text {edd }}$ trends in black hole X-ray Binaries (XRBs).

Throughout this work we assume $\Omega_{m}=0.3, \Omega_{\Lambda}=0.7$, and $H_{0}=70 h \mathrm{~km} \mathrm{~s}^{-1} \mathrm{Mpc}^{-1}$.

\section{THE CHAMP-BASED LLAGN SAMPLE}

The sample of LLAGN employed in this study is obtained by cross-matching the SDSS DR4 spectroscopic sample of galaxies with the X-ray detected sources identified as part of the Chandra Multiwavelength Project (ChaMP). ChaMP is a wide-area serendipitous X-ray survey based on archival X-ray images of the $|b|>20$ deg sky, obtained with Chandra's AXAF CCD Imaging spectrometer (ACIS). A summary of the survey is presented in Green et al. (2004) and Green et al. (2009), while ChaMP results and data can be found at http://hea-www.harvard.edu/CHAMP. The X-ray analysis extends to a total of 392 fields through Chandra Cycle 6, that cover a total of $\sim 30 \mathrm{deg}^{2}$ of sky area.

We limit the investigation to the DR4 dataset in order to employ the measurements of absorption and emission line fluxes and equivalent widths (EW) drawn from the catalog built by the MPA/JHU collaboration ${ }^{5}$. Here the line emission component is separated and subtracted from the total galaxy spectrum based on fits of stellar population synthesis templates (Tremonti et al. 2004). The catalog does not include broad-line objects. To relate the central $\mathrm{BH}$ accretion activity to the host properties, we employ stellar masses of galaxies and the $\mathrm{H} \delta_{A}$ Balmer absorption-line index as a proxy for the age of the associated stellar population, as calculated and presented by Kauffmann et al. (2003b). A detailed analysis of these properties, and their relation to the AGN phenomenon revealed through optical signatures, are presented in Kauffmann et al. (2004).

The cross-match of all ChaMP sky regions imaged by ACIS with the SDSS DR4 spectroscopic footprint results in a parent sample of 15955 galaxies on or near a chip, and a subset of 199 sources that are X-ray detected. Among those, only 107 sources have an off-axis angle (OAA) $\theta<0.2$ deg. and avoid $c c d=8$ due to high serial readout noise; these objects comprise the main sample we employ for this study. Subsequent subsections present details of the X-ray spectral analysis, together with a presentation of their general optical and X-ray properties, the definition of the subsamples based on their optical spectral properties, and a discussion of the selection effects associated with these samples.

\subsection{X-ray spectral analysis}

\footnotetext{
${ }^{4}$ i.e., the shape of the spectral energy distribution quantified by the photon index $\Gamma$ that best fits a power-law $N(E) \propto E^{-\Gamma}$

5 publicly available at http://www.mpa-garching.mpg.de/SDSS/ (Brinchmann et al. 2004)
}

Chandra imaging with ACIS provides energy resolution sufficient to constrain the X-ray spectral properties as well. To characterize the X-ray activity of the ChaMPSDSS galaxies included in our sample, we perform direct spectral fits to the counts distribution using the full instrument calibration, known redshift and Galactic $21 \mathrm{~cm}$ column ${ }^{6} N_{H}^{G a l}$. Source spectra are extracted from circular regions with radii corresponding to energy encircled fractions of $\sim 90 \%$, while the background region encompasses a $20^{\prime \prime}$ annulus, centered on the source, with separation $4^{\prime \prime}$, from the source region. Any nearby sources are excised, both from the source and the background regions.

The spectral fitting is done via yaxx ${ }^{7}$ (Aldcroft 2006), an automated script that employs the CIAO Sherpa ${ }^{8}$ tool. Each spectrum is fitted in the range $0.5-8 \mathrm{keV}$ by two different models: (1) a single power-law plus absorption fixed at the Galactic $21 \mathrm{~cm}$ value: (model "PL"), and (2) a fixed power-law of photon index $\Gamma=1.9$ plus intrinsic absorption of column $N_{H}$ (model "PLfix"). These fits use the Powell optimization method, and provide a robust and reliable one-parameter characterization of the spectral shape for any number of counts. Spectra with less than 100 net counts were fit using the ungrouped data with Cash statistics (Cash 1979), while those with more than 100 counts were grouped to a minimum of 16 counts per bin and fit using the $\chi^{2}$ statistic with variance computed from the data. For the (9) objects with more than 200 counts we employ a third model where both the slope of the power-law and the intrinsic absorption are free to vary (model "PL_abs").

Many of the X-ray detected galaxies in our sample have relatively few net counts (mean 76 , median 19). In such cases, instrumental hardness ratios is often used, in the belief that genuine spectra fitting is not warranted by the data quality. We stress however that spectral fitting provides the most consistent and robust estimates of the physical parameters of interest, the power law slope and intrinsic absorption. Because the ChaMP X-ray exposures span a variety of intervening Galactic columns, include data from both back- and front-side ACIS CCDs, and span 6 years of observations, the spectral response between sources varies significantly. While constraints from spectral fitting may not be tight for low count sources, use of unbinned event data and the appropriate response gives an optimal and unbiased estimate of the fit parameters and their uncertainties, especially important when absorption may be present at different redshifts. Classical hardness ratio analysis on the other hand amounts to grouping the data into two rather arbitrary bins, introducing potential biases and statistical complexity. Interpreting the hardness ratio value for ChaMP sources in disparate fields requires incorporating the instrument response in any case, so we strongly prefer spectral fitting. Nevertheless, when there is only one free parameter, only the overall spectral shape is constrained.

This simple parametrization proves generally sufficient to model the $0.5-8 \mathrm{keV}$ spectra of these objects. Com-

6 Neutral Galactic column density $N_{H}^{G a l}$ taken from Dickey \& Lockman (1990) for the Chandra aimpoint position on the sky.

7 http://cxc.harvard.edu/contrib/yaxx

8 http://cxc.harvard.edu/sherpa 
parisons of $0.5-8 \mathrm{keV}$ fluxes $f_{x}$ (and luminosities $L_{X}$ ) obtained from the PL and PLfix models show good agreement, for the whole sample of galaxies, the average (median) of the difference in these values being $0.07(0.01)$ dex. We caution that the simple power-law fits we use here could be misleading for objects where the absorption is complex (i.e., a partial covering, with one or more absorber potentially being ionized). However, the data quality is insufficient to show that the situation is more complex than a simple power-law.

We compile a set of "best" measurements for $\Gamma$ or $N_{H}$, by using the values obtained from the PL (intrinsic $N_{H}$ fixed at zero) and PLfix ( $\Gamma$ fixed at 1.9) models, respectively. For objects with more than 200 counts we use the $\Gamma$ and $N_{H}$ values obtained from the PL_abs. The mean $\Gamma$ for the whole sample of 107 galaxies is $2.03 \pm 1.38$, with a median of 2.04. The level of intrinsic absorption is generally low. More than $85 \%$ of the sample exhibits $N_{H}<1 \times 10^{22} \mathrm{~cm}^{-2}$, while for $60 \%$ of the objects the spectral fits are consistent with zero intrinsic absorption. Note that, given the simplified model used in fitting the $\mathrm{X}$-ray spectra, these values might not necessarily represent the true distribution of absorption in these objects. Individual measurements of all of these X-ray properties, together with their observational parameters, like the total number of X-ray counts, the exposure time, the off-axis angle, together with their corresponding X-ray source ID, are listed in Table 1 for all 107 objects.

Contribution from thermal emission is expected for some of the objects included in this sample of nearby galaxies, whether or not they show line emission activity. Such a component may provide a reasonable contribution to the total (X-ray) emission even in objects where the dominant ionization mechanism, as identified optically, is a compact nuclear source, i.e., an AGN. LINERs, for instance, have frequently been associated with photoionization by hot, young stars (Filippenko \& Terlevich 1992; Shields 1992; Barth \& Shields 2000), clusters of planetary nebula nuclei (Taniguchi, Shiova, \& Muravama 2000), or more recently (and perhaps more consistent with their older stellar populations), by hot post-AGB stars and white dwarfs (Stasinka et al. 2008).

We attempted fitting the $0.5-8 \mathrm{keV}$ spectra with a Raymond-Smith (R-S) thermal plasma model, with the abundance fixed at 0.5 solar. The choice of abundance level is inspired by previous investigations of LLAGN, e.g., Ptak et al. (1999) and Terashima et al. (2002), even though in many cases the abundance remains poorly constrained. This R-S model fit results seem physically feasible for only about the third of the sample. Reasonable values, in agreement with previous findings, i.e., $k T \lesssim 2 \mathrm{keV}$ are only found for 35 sources. For another third the best fit $k T>10 \mathrm{keV}$. We discuss in more detail the results of fitting this model as a function of the optical spectroscopic properties of these sources in Section 2.5 .

As probably expected, it is the passive galaxies that show the softest power-law slopes we measure $(\Gamma>3.5)$, suggesting that, for these cases in particular, a powerlaw representation may be incorrect. If we instead fit a Raymond-Smith model, we derive reasonable typical temperatures near $\sim 0.7 \mathrm{keV}$. Nonetheless, since even these objects are likely to have some power-law contribution from X-ray binaries, the R-S model is perhaps no better a characterization of the true spectrum than a power-law. The X-ray flux distribution we derive from the R-S model fits to passive galaxies is not significantly different $( \pm 25 \%)$, so we prefer to retain the power-law fits everywhere to facilitate more direct comparison of the different spectral classes.

\subsection{The Optical Spectral Classification}

We identify and classify accretion sources and other types of active systems in both the parent galaxy sample and the X-ray detected subsample, based on their optical emission line properties. It has been argued (Ho, Filippenko, \& Sargent 1997a; Constantin \& Vogeley 2006; Kewley et al. 2006) that the best way to separate accretion sources from starbursts or other types of active systems is via a set of three diagnostic diagrams, which employ four line flux ratios: $\left[\begin{array}{ll}\mathrm{O} & \mathrm{III}\end{array}\right] \lambda 5007 / \mathrm{H} \beta,\left[\begin{array}{ll}\mathrm{N} & \mathrm{II}\end{array}\right] \lambda 6583 / \mathrm{H} \alpha$, [S II] $\lambda \lambda 6716,6731 / \mathrm{H} \alpha$, and $[\mathrm{O} \mathrm{I}] \lambda 6300 / \mathrm{H} \alpha$. Thus, for both samples, we first select a subset of strong emissionline sources that show significant emission in all six lines used in the type classification $(\mathrm{H} \alpha, \mathrm{H} \beta,[\mathrm{O}$ III],[N II], $[\mathrm{S} \mathrm{II}]$, and $[\mathrm{O} \mathrm{I}]$ ), and a set of passive objects that show insignificant line emission activity. An emission feature is considered to be significant if its line flux is positive and is measured with at least $2 \sigma$ confidence. Following Kewley et al. (2006) classification criteria, the emissionline objects are separated into Seyferts (Ss), LINERs (Ls), Transition objects (Ts), and star-forming, or H II galaxy nuclei.

This method of classifying low luminosity actively lineemitting galaxy nuclei has the disadvantage that it leaves unclassified a high fraction $(\sim 40 \%)$ of galaxies, which show strong emission features, but not in all six lines considered here. The condition for strong emission in [O I] in particular is significantly restrictive. Moreover, another quite large $(\sim 25 \%)$ fraction of the emission-line objects remains unclassified, as their line ratios, although accurately measured, do not correspond to a clear spectral type in all three diagrams. In the majority of such cases, while the $[\mathrm{N} \mathrm{II}] / \mathrm{H} \alpha$ ratio shows relatively high, $\mathrm{S}$ like values, the corresponding $[\mathrm{S} \mathrm{II}] / \mathrm{H} \alpha$ and/or $[\mathrm{O} \mathrm{I}] / \mathrm{H} \alpha$ place them in the $\mathrm{T}$ or $\mathrm{H}$ II -like object regime; thus, because the $\left[\begin{array}{ll}\mathrm{S} & \mathrm{II}\end{array}\right]$ and $\left[\begin{array}{ll}\mathrm{O} & \mathrm{I}\end{array}\right]$ emission lines are better AGN-diagnostics than [N II], these systems are likely to be excluded from the AGN samples selected via these classifications. As a consequence, our samples based on the 6 -line classification are small.

To enlarge our samples of galaxy nuclei of all spectral types, we also explored an emission-line classification based on only the $[\mathrm{O}$ III $] / \mathrm{H} \beta$ vs. $[\mathrm{N}$ II $] / \mathrm{H} \alpha$ diagram, i.e., a 4-line classification method, for the $\mathrm{X}$-ray detected sources. The emission line galaxy samples comprise thus all objects showing at least $2 \sigma$ confidence in the line flux measurements of these four lines only. The delimitation criteria of H II's and T's remain unchanged, while Ss and Ls are defined to be all objects situated above the Kewley et al. (2006) separation line, and with $[\mathrm{O}$ III $] / \mathrm{H} \beta>3$ and $<3$ respectively. The 4 -line and 6-line classifications result in significantly different classes when applied to optically selected galaxies in general (Constantin \& Vogelev 2006). In particular, true properties become heavily blended into the dominant population of LINERs (or Ts, depending on the sepa- 
ration lines used in the diagnostic diagrams). Interestingly, however, when applied to the X-ray sample, the 4 -line classes fall well within the 6 -line loci; although Ss and Ls are separated only by their $[\mathrm{O} \mathrm{III}] / \mathrm{H} \beta$ line flux ratio, they remain clearly separated into the $[\mathrm{S} \mathrm{II}] / \mathrm{H} \alpha$ and $[\mathrm{O} \mathrm{I}] / \mathrm{H} \alpha$ diagrams as well. Figure 1 shows how the 6-line (top) and the 4-line (bottom) classifications compare for the ChaMP X-ray detected galaxies.

Although the sample of X-ray detected galaxies is small, this comparison indicates that adding X-ray detection makes the 4-line classification more secure, and that the need for the (usually unavailable) $[\mathrm{O}$ III $] / \mathrm{H} \beta$ vs. $[\mathrm{S} \mathrm{II}] / \mathrm{H} \alpha$, and $[\mathrm{O} \mathrm{III}] / \mathrm{H} \beta$ vs. $[\mathrm{O} \mathrm{I}] / \mathrm{H} \alpha$ diagrams is not as stringent as in the cases where only optical information is available. We will thus consider for the analysis presented in this paper only the 4-line classification.

\subsection{The X-ray detection fraction of the LLAGN}

The "cleaning" role of the X-ray detection in finding and defining LLAGN is even more obvious when we compare the fractions of X-ray detected objects by spectral type, both relative to the parent sample of nearby optically selected objects and the subsample of X-ray detected galaxies. Table 2 lists these percentages, where for the parent optically selected sample we consider only the SDSS galaxies on ACIS chips (excluding chip S4, $c c d \# 8$, and with off-axis angle $\theta<0.2 \mathrm{deg}$, consistent with the conditions used in compiling the X-ray samples. The first two columns show the number (and fraction) that each spectral type represents, among (1) the optical parent sample and (2) the X-ray-detected subsample. The third column lists the raw fraction of X-ray detections per spectral type.

That the X-ray detection is very efficient in finding LLAGN, particularly Seyferts, is quite apparent. While narrow-line Ss usually make up only $<2-4 \%$ (Ho. Filippenko, \& Sargent 1997b; Constantin \& Vogelev 2006) of the optically selected nearby galaxies, the X-ray detection increases the chances of finding them tenfold. Ts and Ls, where an AGN contribution to the total ionization power is expected, are also much better represented in the $\mathrm{X}$ ray detected sample of galaxies, their fractions being $\sim 3 \times$ larger than when only optical selection is employed. Some $65 \%$ of the optically defined Ss are detected in X-rays, while the other spectral types hardly reach an X-ray detection fraction of $20 \%$. Ls are the second most X-ray active sources within nearby galaxies, while Ts and the galaxies that show some/weak emission line activity account for less than $1 / 5$ th of the sample. As we discuss further in Section 2.5, only the luminous HiIs are detected in X-rays. While the X-ray detection rate of the $\mathrm{H}$ IIs is basically consistent with zero, when detected, their X-ray emission is moderately strong, $L_{X}=10^{39}-2.5 \times 10^{41} \mathrm{erg} \mathrm{s}^{-1}$; half of the $\mathrm{H}$ II detections show X-ray luminosities higher than the level that can be reached without contribution from AGN $\left(10^{40} \mathrm{erg} \mathrm{s}^{-1}\right)$, suggesting once more that the nuclear emission in these sources might not be completely driven by stellar processes.

The only previous studies that encompass the whole spectral variety of LLAGN are Roberts \& Warwick (2000) and Parejko et al. (2008), which employ ROSAT (HRI and RASS respectively). Their search for X-ray emitting nearby galaxy nuclei, optically characterized via the Palomar and SDSS surveys respectively, concluded in soft X-ray detection rates of $\sim 70 \%$ of both Ss and Ls (HRI), $\sim 70 \%$ of Ss and $\sim 60 \%$ Ls (RASS), $\sim 40 \%$ (HRI) and $<10 \%$ (RASS) of H II's, and $\sim 30 \%$ of passive galaxies (both cases). Comparison of these detection rates to ours is problematic, because of their softer instrument bandpass, lower sensitivity, but wider sky coverage.

Hard X-ray studies of homogeneously selected samples including all spectral types of nearby active galaxies are practically non-existent. Ls, and particularly those found in the Palomar survey, have been clearly privileged in terms of X-ray targeting (e.g.. Ho et al. 2001). Their detection rates are found to be significantly higher than what we report here based on the serendipitous ChaMP survey. Ho et al. (2001) reports $\mathrm{a} \sim 70 \%$ detection rate, while, when chosen for having a flat-spectrum radio core, Ls are found to be $100 \%$ Xray active (Terashima \& Wilson 2003). Later studies claiming better accounting for selection and classification as LINER (Satyapal et al. 2004; Dudik et al. 2005; Pellegrini 2005; Satvapal et al. 2005; Flohic et al. 2006; Gonzalez-Martin et al. 2006) conclude with lower fractions, $\sim 50 \%$.

ChaMP has the distinct advantage of presenting a large, homogeneous serendipitous sample of LLAGN. The detection fraction is not an intrinsic property of galaxies, but rather a convolution of galaxy properties with optical survey depth, and the X-ray sensitivity vs. sky area curve. As described in (Green et al. 2009), the ChaMP is characterising the X-ray sensitivity at the position of every SDSS galaxy, which will enable us to compile the unbiased fraction of galaxies by optical spectral type (e.g., Ls) that fall in X-ray luminosity bins, from sky volumes complete to those limits. We will present the results of such an investigation in a subsequent paper.

\subsection{Selection Effects: the ChaMP X-ray Galaxy Sample is Minimally Biased}

$\mathrm{X}$-ray and optical emission are correlated. Thus, while the X-ray selection is one of the most powerful tools to exploit in detecting accretion sources, it is also expected that this selection picks up, selectively, the brightest (optical) sources. Due to its serendipitous character, ChaMP should however reduce such effects. Note that, out of $107 \mathrm{X}$-ray detections that this ChaMP sample of galaxies provides, only 13 are targets.

We explore in Figure 2 the biases that X-ray detection potentially adds to our sample. Comparisons of the distributions of redshift $z$, apparent and absolute $r$-band (SDSS) magnitudes, $r$ and $M_{r}$ respectively, and of the concentration index $C^{9}$ as a proxy for the morphological type of these galaxies. Both for the whole optically and X-ray selected samples (histograms on the left panel) and separately per spectral type (the right panel) show, pleasingly, that biases are not strong.

However, $\mathrm{H}$ II galaxies are of lower $z$ and brighter $M_{r}$ when detected in X-ray. As shown in the next section, the $\mathrm{H}$ IIs are generally weak X-ray sources. The tendency for X-ray detected galaxies to appear brighter in appar-

${ }^{9} C=R_{50} / R_{90}$, where $R_{50}$ and $R_{90}$ are the radii from the center of a galaxy containing $50 \%$ and $90 \%$ of the Petrosian flux 
ent magnitude $(r$, by $\sim 0.5 \mathrm{mag})$ seems to be caused by the large difference in $r$ between the HII galaxies alone, as all the other types of sources show very similar ranges, averages or medians, when analysed separately.

The concentration index $C$ appears to be somewhat larger for the X-ray objects. Several factors are likely to account for this. The H IIs are the least concentrated optically, and have the lowest X-ray detection fraction. X-ray detection is sensitive to the AGN activity, which is more prevalent in the early type (massive bulgedominated) galaxies (e.g.. Ho, Filippenko, \& Sargent 2003; Kauffmann et al. 2004). Nuclear activity is also expected to increase $C$ simply by adding light to the core. Note that there is no significant difference in this parameter in regard to Ss' X-ray selection. However, Ss make up for a tiny fraction of $z \approx 0$ galaxies, and the morphology of their hosts spans quite a range.

\subsection{General X-ray Properties in Relation to Optical Spectroscopic Classification}

X-ray information about the nearby galaxy centers constrains the contribution of accretion-related processes to their optical spectral characteristics. We present in this section the distributions of a variety of X-ray properties for the whole sample as well as for subsamples by optical spectral class. Figure 3 shows the distributions of the X-ray counts, the $0.5-8 \mathrm{keV}$ unabsorbed X-ray fluxes, the best-fit X-ray photon indices $\Gamma$ and the intrinsic neutral hydrogen column densities $N_{H}$. These measurements are shown for the whole sample of X-ray detected nearby galaxy nuclei (left column), and separately per spectral type (right column).

For X-ray fluxes, we show the values derived using the primary power-law fitting models discussed in Section 2.1 (1) a single power-law with no intrinsic absorption and $(2)$ a fixed power-law $(\Gamma=1.9)$ with absorption. The $f_{x}$ values are generally consistent with each other for the whole range of brightness and optical spectral type. As expected, the X-ray brightest objects are among Ss, however, even for this spectral type the range of values remains pretty broad, spanning 3 orders of magnitude. Note also that the few $\mathrm{H}$ II galaxies that are X-ray detected are in general brighter than the passive systems.

In terms of the X-ray spectral shape, the ChaMP nearby galaxies are quite a diverse population. The mean photon index per optical spectral type shows however a rather clear dependence on the spectral type: Ss show the hardest $0.5-8 \mathrm{keV}$ spectral shape, becoming softer and softer from Ts to Ls to the Passive galaxies which are clearly the softest. The H II galaxies are unexpectedly hard in average $\Gamma=1.46$, however, two particularly hard detections clearly weight the subsample in this direction. The $T$ s and Ls average at $\Gamma \approx 2$.

The $N_{H}$ values are poorly constrained for this sample, and there is no obvious correlation with the optical spectral type. It is however obvious that the Ss are the sources with the highest fraction of non-zero absorption. Since all our Ss are of type 2, i.e., lack broad emission lines in their spectra, the unification paradigm predicts that many will show signature of absorption in X-rays. A typical unabsorbed power-law $\Gamma=1.9$ requires a column density of $N_{H}=8 \times 10^{21} \mathrm{~cm}^{-2}$ to yield an apparent $\Gamma=1$ similar to the mean value found for our S subsample, which is consistent with the observed mean $N_{H}$ for these particular systems.

\section{PROBING THE SEQUENCE}

The $H$ II $\rightarrow S / T \rightarrow L$ evolutionary sequence proposes a comprehensive picture for the co-evolution of AGN and their host galaxies. This scenario is supported by and strengthens previous studies of AGN, starformation activity and their co-evolution in nearby galaxies (Kauffmann et al. 2004; Heckman et al. 2004), and may enhance our understanding of how AGN work and evolve in relation to both their hosts and their environments (Constantin. Hoyle, \& Vogeley 2008).

$\mathrm{X}$-rays, as primary signatures of supermassive $\mathrm{BH}$ accretion, offer a critical verification of the proposed sequence. $L_{X}$ and spectral fits characterize the sequence in terms of strength and mode of accretion, especially the order of $S \mathrm{~s}$ and $T$ s within the $H$ II $\rightarrow$ $S / T \rightarrow L$ cycle. Both the bulge nebular properties, and the small and large scale environments of $S$ s and $T$ s, are very similar and remain intermediate between those of $\mathrm{H}$ IIs and $L \mathrm{~s}$. The only parameters showing a "jump" in the otherwise smooth trends are $\mathrm{H} \alpha / \mathrm{H} \beta$ Balmer decrements and the nearest neighbor distance $d_{1 \mathrm{nn}}$ (Constantin. Hoyle, \& Vogeley 2008). $\mathrm{H} \alpha / \mathrm{H} \beta$ provides a measure of absorption, and perhaps also the amount of fuel available for accretion, which we can now test directly against both $L_{X}$ (accretion power) and Xray spectral constraints.

We present in Figure 4 a comparison of the degree to which optically and X-ray selected galaxies follow the proposed sequence in terms of the black hole mass $M_{\mathrm{BH}}$, obtained via $\sigma_{*}$ measurements and the $M_{\mathrm{BH}}-\sigma_{*}$ relation (Tremaine et al. 2002), the (dust corrected) stellar mass $M^{*}$ (Kauffmann et al. 2003a), the Balmer decrement $\mathrm{H} \alpha / \mathrm{H} \beta$ as a proxy for dust extinction, the $\mathrm{H} \delta_{A}$ Balmer absorption index as a measure of the age of the associated stellar population, $L[O I I I]$, and the accretion rate expressed as $L / L_{\text {edd }}$, where $L=L_{\text {bol }}=600 \times L[O I I I]$ for the bolometric correction (Heckman et al. 2004; Kauffmann \& Heckman 2009), and the $L[O I I I]$ is extinction-corrected using the corresponding Balmer decrements and a $\tau \propto \lambda^{-0.7}$ attenuation law (Charlot \& Fall 2000). Given the (spectral) definition of passive galaxies (i.e., lacking optical emission line activity), there are no measurements of the Balmer decrement, $L[O I I I]$ and $L / L_{\text {edd }}$ for these sources. It is notable that in all measures, and for all types of sources, the X-ray and optically selected sources are very similar, and appear to obey the $S \rightarrow T \rightarrow L \rightarrow$ $P$ sequence.

If anything, the sequence appears stronger among the $\mathrm{X}$-ray selected galaxies, both in median/average values and in their distributions of individual measurements, which span smaller ranges of values than for the optically selected objects. Among the weakest accreting objects, Ls and Passive galaxies, the X-ray selection tends to pick up more massive objects, with heavier BHs, and older stellar populations; there is however no obvious difference in these parameters for the other types of sources. As expected, these massive systems also appear to have smaller [O III] luminosities and accretion rates, accentuating the sequential $S$ to $T$ to $L$ drop in these parameters suggested by optically selected/defined objects. Also, while the Balmer decrement distributions suggest that, 
in general, the X-ray selection is not strongly affected by dust, the slightly more obscured X-ray detected H IIs and less obscured X-ray detected Ls make the sequence more apparent.

Figure 5 illustrates how the $0.5-8 \mathrm{keV}$ X-ray luminosity $L_{X}$ and the corresponding $L / L_{\text {edd }}$ behavior along the $S \rightarrow T \rightarrow L \rightarrow P$ sequence. For the sake of comparison, we show $L_{X}$ values obtained via two spectral fitting models, 1 . a power-law with no intrinsic absorption (only the Galactic one) and 2. a fixed power-law with $\Gamma=1.9$ and with variable intrinsic absorption added to the Galactic level. The sequence is supported by both types of measurements, albeit stronger when the simple power-law model is used. As discussed in Sections 2.1 and 2.5. the free power-law with no intrinsic absorption spectral model, that seems to best characterize this sample of objects, makes the statistical sequence even more probable. For the sequence in the accretion rate, we calculate $L / L_{\text {edd }}$ using $L / L_{X}=16$ for the bolometric luminosity, as suggested by $\mathrm{Ho}$ (2008), and the $\mathrm{BH}$ masses estimated from their host stellar velocity dispersion $\sigma_{*}$ using Tremaine et al. (2002). We also contrast the Xray measurements with those where $L_{\text {bol }}$ derived from $L[O I I I]$; note that while $L_{X}$ and the derived $L / L_{\text {edd }}$ are available for the whole sample of 107 objects, only 69 of them exhibit strong $2-\sigma$ detectable, [O III] line emission. The $S \rightarrow T \rightarrow L \rightarrow P$ galaxy sequence compares well in both optical and X-ray measurements. The values of all these parameters decrease monotonically in both median and average values, from Ss to Ts, to Ls and Passive galaxies, consistent with what optical properties of these sources put forward.

The $\mathrm{H}$ IIs are the only apparent exception here. The small number statistics for these galaxy nuclei preclude any strong conclusions. Given the expected high sensitivity to soft sources that these measurements provide, their spectra tend to be on the hard side. Obscuration is a notorious cause for spectral hardening, and thus, the possibility that these objects hide in their centers obscured $\mathrm{BH}$ accretion is still not ruled out.

Note also that the power-law slope $\Gamma$ that best fits the $\mathrm{X}$-ray spectra increases from Ss to Ts, to Ls, in both median and average values, showing a tendency of softening of the spectra from Ss to passive galaxies along the proposed sequence (Figure 3). This is quite an interesting finding, as in other AGN, mostly the luminous type 1 AGN, observations suggest an opposite trend: the stronger accreting (and more luminous) sources are the softer ones, most recently quantified by Kelly et al. (2008); Shemmer et al. (2008). It is interesting to note that a spectral softening with strengthening of the accretion process/rate is also a generally common feature of the X-ray Binaries (XRBs) with reasonably high $L / L_{\text {edd }}$ (e.g.. Kubota \& Makishima 2004). Note however that, when the Eddington ratio is less than a critical value, $L / L_{\text {edd }} \lesssim 0.01$, i.e., XRBs are observed in their low/hard state, there is a clear trend for softening with further weakening of the accretion rate (Kalemci et al. 2005; e.g. Yamaoka et al. 2005; Yuan et al. 2007). We investigate this finding in more detail in the following section, and discuss the analogy with the XRB phenomenon.
Investigations of how X-ray parameters depend on the accretion rate relative to the Eddington rate are expected to offer important constraints on physical models of the AGN X-ray emitting plasma, particularly its geometry. A hot, optically thin corona that Compton up-scatters UV photons from the optically thick disk (Shakura \& Syunyaev 1973; Haardt \& Maraschi 1991) seems to fit reasonably well the X-ray emission of the highly accreting systems, particularly when it is associated with a hot, possibly patchy and "skin"-like structure "sandwich"-ing the cold disk (Navakshin 2000; Czerny et al. 2003). Other geometries remain however viable, among them an accretion disk evaporating into a hot inner flow (Shapiro et al. 1976; Zdziarski et al. 1999), or combinations of a hot inner flow and the patchy corona (Poutanen et al. 1997; Sobolewska et al. 2004). For LLAGN, with $L / L_{\text {edd }}<10^{-9}-10^{-5}$, the accretion flow has been hypothesized to originate from a geometrically thick and hot disk-like structure that is inefficient at converting gravitational potential energy into radiation, the radiatively inefficient accretion flow (RIAF) model (e.g., Naravan \& Yi 1994; Blandford \& Begelman 1999; Naravan et al. 2000). Models suggest that there is a transition/switch from a standard disk to an advection dominated accretion flow (ADAF; or, a radiatively inefficient accretion flow, RIAF) when $L / L_{\text {edd }}$ declines below a critical value within a certain transition radius (Esin et al. 1997; Yuan \& Naravan 2004; Lu et al. 2004). In either case, radiation pressure driven outflows can also alter the physics of the corona (Proga 2005). Because the efficiency in producing an X-ray accretion flow and in driving the outflows depends on the $\mathrm{BH}$ mass and its accretion rate, it is important to understand the interdependence of these parameters on the X-ray properties, particularly the shape of the X-ray spectrum, i.e., the $\mathrm{X}$-ray photon index $\Gamma$.

The relationship between $\Gamma$ and the Eddington ratio $L / L_{\text {edd }}$ is relatively well studied, and yet a controversial issue. These two parameters seem to be positively correlated for objects accreting at relatively high Eddington ratios, i.e., quasars, luminous type 1 Seyferts (Kelly et al. 2008; Shemmer et al. 2008), while for low $L / L_{\text {edd }}$ values the situation remains uncertain, mainly due to the lack of quality data in that regime. The conclusion so far seems to be that the shape of the hard X-ray power-law is largely controlled by $L / L_{\text {edd }}$. For the luminous, strongly/efficiently accreting sources, it is proposed that the corona acts as a "thermostat" by (Compton) cooling more efficiently when the disk emission increases, producing more soft photons, and thus steepening the hard X-ray spectrum. This scenario also accounts nicely for the generally narrow range of $L / L_{\text {edd }}$ and $\Gamma$ values measured in (optically selected) quasars or luminous AGN, in general $\left[L / L_{\text {edd }} \sim 0.3\right.$ with a typical dispersion of a factor of 5; (McLure \& Dunlop 2004; Kollmeier et al. 2006; \begin{tabular}{l|l|l}
\hline Netzer et al. & $2007 ;$ & Shen et al. 2008). $\Gamma \sim 1.5-$
\end{tabular} 2.5; (Vignali et al. 2005; Shemmer et al. 2008)]. For LLAGN, the $\Gamma-L / L_{\text {edd }}$ relation remains only vaguely constrained.

\section{1. $\Gamma-L / L_{\text {edd }}$ relation for nearby $A G N /$ galaxies


The relation between the X-ray photon index $\Gamma$ and the Eddington ratio for the ChaMP X-ray detected galaxies is illustrated in Figure 6. The Eddington luminosity $L_{\text {edd }}$ is calculated as indicated in Section 3. using $M_{\mathrm{BH}}$ values estimated based on stellar velocity dispersion $\sigma_{*}$ via Tremaine et al. (2002), while the bolometric luminosity is calculated based on $L_{X}$, using the average bolometric correction of $L_{\mathrm{bol}} / L_{X}=16$ (Ho 2008). There is a rather clear trend of spectral hardening with increasing accretion rate. $\Gamma$ and $L / L_{\text {edd }}$ are found to be negatively correlated.

Defining an accretion rate, i.e., calculating $L / L_{\text {edd }}$, for galaxies with $L_{X}<10^{42} \mathrm{erg} \mathrm{s}^{-1}$ may be misleading if the $\mathrm{X}$-ray emission in these objects is dominated by X-ray sources other than an accreting super massive BH (e.g., individual compact binaries or hot diffuse gas). Note however that the $x$-axis of Figure [6] is simply the measure of $L_{X} / M_{\mathrm{BH}}$ (or better, $L_{X} / \sigma_{*}{ }^{4}$ ), where both $L_{X}$ and the $\mathrm{BH}$ mass $M_{\mathrm{BH}}\left(\right.$ or $\left.\sigma_{*}\right)$ are measured in exactly the same manner for all of the objects involved, and the trend remains even if this parameter is not interpreted as an accretion rate. The strongest likely dilution to accretion emission comes from contributions of the hot ISM in passive galaxies. Hot gas emission is soft, and relatively stronger in more massive hosts, both of which would push the passive galaxy points toward softer X-ray spectra (larger $\Gamma$ ) and lower $L_{X} / M_{\mathrm{bh}}$, which might spuriously accentuate the observed trend even in the absence of significant accretion power. Indeed, the observed trend is weakened once the passive galaxies are removed (Table 4). So while we rule out significant extended emission contributions in this sample, more detailed examination of such objects is warranted to determine the relative fractions of nuclear vs. extended hot gas contributions.

We measure the significance of the $\Gamma-L / L_{\text {edd }}$ anti-correlation using the Spearman-rank test. The Spearman-rank coefficient, the chance probability, and the number of sources for each correlation are listed in Table 3. We fit the anti-correlation points with a linear least-squares method for the whole sample and for the subsamples of galaxies corresponding to different spectral types, using the mpfit ${ }^{10}$ routine, being able to account for the errors in $\Gamma$. We show, for comparison, both the error weighted (continuous line) and the unweighted (dotted line) best-fits in Figure 6]. The scatter around the best linear fit is large, and as expected the points with the largest error bars show the largest deviation. However, within the errors, the results of the fit remain unchanged when we use only objects with small measurements errors (i.e., $\Delta \Gamma / \Gamma<50 \%$ ). The results for the linear regression coefficients and the corresponding $\chi^{2}$ and dof values are listed in Table 4. For the sake of considering "cleaner" AGN-like activity only, we also list here the results of such a fitting techniques to samples that exclude the Passive galaxies and the $\mathrm{H}$ IIs, and for samples of luminous X-ray systems $\left(L_{X} \gtrsim 10^{42} \mathrm{erg} \mathrm{s}^{-1}\right)$ only, and indications of an anticorrelation, albeit weaker, remain.

The linear regression fits might appear at odds with the conclusion of the Spearman test, which indicates that $\Gamma$ and $L / L_{\text {edd }}$ are possibly anticorrelated for all the subsamples presented here. Note however that such a dis-

10 http://www.physics.wisc.edu/c̃raigm/idl/fitting.html crepancy appears for the subsamples where the Spearman test remains rather inconclusive as, the probability that an anticorrelation appears by chance is large. Moreover, the Spearman test ignores the errors, and thus tests the unweighted data, for which linear regression fits are always consistent with negative slopes. It is clear however that for Seyferts in particular, there is no evidence for either positive or negative correlation between $\Gamma$ and $L / L_{\text {edd }}$. This has been seen in other samples as well (Winter et al. 2009), and it is an important result. Nevertheless, investigations of the LLAGN phenomenon should not be restricted to these types of sources only.

In an attempt to provide some more physical insights into the reality and significance of this new trend, we also explore here the possible correlations between various $\mathrm{X}$-ray measures that might influence (if not artificially create) it. In particular, we scrutinize the way our measured $\Gamma$ relates to the number of counts, the X-ray flux and luminosity. Some studies showed that the photon index correlates with the X-ray luminosity, becoming softer in more luminous sources, whether measured in soft, $0.2-2 \mathrm{keV}$ (Forster \& Halpern 1996; Lu \& Yu 1999; Gierlinski \& Done 2004; Williams, Mathur, \& Pogge 2004), or hard, $2-10 \mathrm{keV}$ (Dai et al. 2004; Porquet et al. 2004; Wang. Watarai \& Mineshige 2004) bands only, or comprising the whole spectrum, and even as they vary (Chiang et al. 2000; Petrucci et al. 2000; Vaughan et al. 2001). However, because many others have not found such trends, the idea that the choice of the sample involved in these studies may contribute to producing the correlations has also been put forward. Interestingly, our ChaMP sample does not show this correlation either. Figure 7 illustrates the dependence of $\Gamma$ measured in the $0.5-8$ $\mathrm{keV}$ range as a function of the total number of counts in this energy range, the X-ray flux, and the X-ray luminosity. As before, we emphasize the different optical spectral classifications; the fact that, e.g., the various types of galaxies separate rather well from each other in diagrams like this suggests that the correlation is physical, and most probably related to the accretion physics in these nuclei, rather than an artificial effect of fitting various (simple) models to their X-ray spectra.

\subsection{Comparison with high z QSOs}

The anti-correlation between $\Gamma$ and $L / L_{\text {edd }}$ (or simply, $\left.L_{X} / M_{\mathrm{BH}}\right)$ that we find to characterize the nuclear emission of nearby galaxies is certainly surprising. This trend is opposite to what more luminous galaxy nuclei, i.e., quasars, exhibit: their X-ray spectra soften as they become more luminous. Figure 8 shows the $\Gamma-L / L_{\text {edd }}$ anti-correlation followed by the low luminosity galaxy nuclei along with measurements of $\Gamma$ and $L / L_{\text {edd }}$ for a sample of SDSS quasars with optical spectra that are ChaMP detected and X-ray analysed in the same manner we handled our sample of nearby galaxy nuclei (Green et al. 2009). The quasar $L / L_{\text {edd }}$ values are obtained using the average bolometric correction of $L / L_{X}=83$ estimated by $\mathrm{Ho}_{\mathrm{O}}$ (2008), with no luminosity-dependence, and the black hole masses from Shen et al. (2008).

To ease comparison with previous work on quasars, we use in this plot the $2-10 \mathrm{keV} L_{X}$ luminosity, which is obtained by extrapolating the measured unobscured $L_{0.5-8 \mathrm{keV}}$ value, using the best $\Gamma$ measurements. Note 
that the anti-correlation between $\Gamma$ and $L / L_{\text {edd }}$ that nearby galaxy nuclei show is even more pronounced when the $2-10 \mathrm{keV} L_{X}$ is plotted against $\Gamma$. This is expected, as softer objects will be less luminous in $2-10 \mathrm{keV}$ than in the $0.5-8 \mathrm{keV}$ range, while the hard objects will be more luminous. The magnitude of this effect, i.e., the ratio of the two $L_{X}$ values is a function of the photon index $\Gamma$, as given by

$$
L_{X}(2-10 \mathrm{keV})=L_{X}(0.5-8 \mathrm{keV}) \times \frac{10^{2-\Gamma}-2^{2-\Gamma}}{8^{2-\Gamma}-0.5^{2-\Gamma}} .
$$

The largest difference, and thus the most significant effect on the shape of the $\Gamma-L / L_{\text {edd }}$ trend, is for the softest $(\Gamma \gtrsim 3)$ objects, however it does not exceed $\sim 1$ dex. Note that for these particular objects the measurement errors are also among the highest, and hence contributed the least weight to the best-fit $\Gamma-L / L_{\text {edd }}$ relation.

The ChaMP quasars fall well within the locus of values expected based on previous work. The ChaMP quasars do not show, however, a clear $\Gamma-L / L_{\text {edd }}$ positive correlation. The ChaMP quasars span a wide range in redshift and their $L / L_{\text {edd }}$ measurements reflect a mix of $\mathrm{BH}$ mass estimates based on all $\mathrm{H} \beta, \mathrm{Mg} \mathrm{II}$, and $\mathrm{C}$ IV, which may add scatter to an underlying correlation (Shen et al. 2008). We show for comparison the results of linear regression fits of the $\Gamma-L / L_{\text {edd }}$ correlation reported by Shemmer et al. (2008) and by Kelly et al. (2008), with the results for the $H \beta$ and C IV based estimates of the $M_{b h}$ shown separately. Note that we use here these data only for the purpose of global comparison of the quasar properties with those of the nearby galaxy nuclei, and do not attempt to improve upon the previous work on the characterization or calibration of the $\Gamma-L / L_{\text {edd }}$ relation for quasars.

Clearly, the quasar $\Gamma$ 's are not negatively correlated with their Eddington ratios, even with the shift in $L_{X}$ produced by the energy conversion mentioned above, which might contribute to such trend (the quasar X-ray data is analysed and measured via the same techniques employed for the nearby galaxy nuclei). Thus, over the whole $L / L_{\text {edd }}$ range we explore here by putting together luminous and weak AGN, there is clearly a break in the $\Gamma-L / L_{\text {edd }}$ correlation, which seems to happen at $L / L_{\text {edd }} \approx 10^{-2}$, and $\Gamma \gtrsim 1.5$. It is important to note that, for the samples of quasars investigated by both Kelly et al. (2008) and Shemmer et al. (2008), the scatter in the claimed correlation between $\Gamma$ and $L / L_{\text {edd }}$ is largest as the samples reach the weakest accretion rates (while hardly reaching the $L / L_{\text {edd }} \approx 10^{-2}$ level), and the hardest values ever measured for the quasar photon in$\operatorname{dex} \Gamma \gtrsim 1.5$; while suggestive of a break, the quasar data alone cannot however be used to single it out. Other studies that include measurements of lower luminosity managed to point out these types of deviations from the expected correlation (e.g.. Zhang, Dultzin \& Wang 2008; Green et al. 2009), however, the data remained sparse at those levels of accretion, leaving the effect unquantified.

We note here that the comparison of the nearby galaxies' central X-ray emission with that of the high redshift QSOs may be inadequate since X-rays from SF activity is not yet accounted for in the former.
We addressed this issue by including in the modelling of the original X-ray spectrum (Section 2.1) a $\Gamma=2$ component with the expected $L_{X}(S F)$. A power-law with $\Gamma=2$ provides the best simple description of the mix of hot gas and high mass Xray binaries (HMXBs) that comprise the SF activity (e.g., Kim et al. 1992a,b; Nandra \& Pounds 1994; Ptak et al. 1999; George et al. 2000; Colbert et al. 2004; Reddy \& Steidel 2004; Lehmer, et al. 2005). To estimate the potential $L_{X}(S F)$ in each galaxy, we use star-formation rates (SFR) calculated and available for these objects in the MPA/JHU catalog (Section 2, Brinchmann et al. 2004), along with the $L_{X}-S F R$ correlation quantified by Gilvanov, Grimm, \& Sunyaev (2004). There are 83 objects with total $L_{X}$ above the line describing the $L_{X}-S F R$ correlation, for which we modelled the remaining - presumably AGN component. This reanalysis produces significant deviations from the $\Gamma-L_{X} / L_{\text {edd }}$ correlation only for $6 \%$ of the sample ( 5 out of the 83 objects involved in this analysis). The scatter and the error bars for both $\Gamma$ and $L_{X}$ are only slightly increased. The slope of the correlation flattens but remains consistent within the errors with our previous measurements.

\subsection{The "break" in the $\Gamma-L / L_{\text {edd }}$ correlation and comparison with XRBs}

The X-ray photon index $\Gamma$ and the Eddington ratio $L / L_{\text {edd }}$ show a double-sloped relation, with positive and negative correlations above and below $L / L_{\text {edd }} \approx 0.01$ and $\Gamma \gtrsim 1.5$ respectively. We see now that, a given AGN spectral index $\Gamma$ may correspond to two different luminosity levels, with the luminosity difference greater for sources characterized by softer spectra (Figure 8). This break in the $\Gamma-L / L_{\text {edd }}$ relation, when studied over a large range of accretion power, fits well into the theoretical ideas of a transition in the AGN accretion mode: a standard (Shakura \& Syunyaev 1973) accretion disk/corona at high Eddington rates, i.e., quasar phase, and an ADAF (e.g.. Naravan \& Yi 1994) at low $L / L_{\text {edd. }}$ This break we find in the $\Gamma-L / L_{\text {edd }}$ relation may provide the best empirical evidence to date for such a transition.

The inflection point in the $\Gamma-L / L_{\text {edd }}$ relation is dominated by $\mathrm{Ts}$, consistent with the optical nature of these systems. While hypothesized to be the result of mixed AGN and SF ionization (e.g.. Ho, Filippenko, \& Sargent 2003; Constantin et al. 2009), their AGN component could be either an S- or L-like. Ss appear to be the low $L$ quasar-like, efficiently accreting systems, while the Ls are the ADAF objects, so T's locus at the break region is suggestive of a switch in the accretion mode. The optically-defined Ts are then transition systems that map the X-ray inflection as well.

Note that this idea of transition in the accretion mode, from ADAF to standard-disk as the accretion rates increases, has been first proposed, and later developed, mainly based on results of investigations of smaller BH mass accretors, i.e., stellar mass black-hole X-ray binaries, or XRBs; see Naravan \& McClintock (2008) for a recent review. Interestingly, the relation between $\Gamma$ and $L / L_{\text {edd }}$ that XRBs exhibit in the different phases of their temporal variability is also multivalued: a given spectral index $\Gamma$ may correspond to two different lumi- 
nosity levels, with the luminosity difference greater for sources characterized by softer spectra. That is, the XRBs show a positive $\Gamma-L / L_{\text {edd }}$ correlation while in their high/soft states (e.g.. Kubota \& Makishima 2004), and an anti-correlation while in their low/hard states (e.g., Yamaoka et al. 2005). Several clear examples of such a turn (or convergence point) in the $\Gamma-L / L_{\text {edd }}$ relation measured in XRBs are illustrated in, e.g., Yuan et al. (2007) and Wu \& Gu (2008).

In terms of the physical phenomenology governing the black hole accretion process over more than 10 orders of magnitude in the Eddington ratio, the transition from an ADAF to a standard disk accretion seems to adequately account for both the XRB and AGN emission properties. The ADAF scenario qualitatively explains the anti-correlation via Comptonization of thermal synchrotron photons as the dominant cooling mechanism at low $L / L_{\text {edd }}$ ratios: the Compton $y$-parameter increases with increasing of the optical depth, which is caused by increase of the accretion rate, the X-ray spectrum becomes harder (Esin et al. 1997). Further increase in the accretion rate would cause both an increase in the released energy and a decrease in the electron temperature, weakening the corona, and consequently the optical depth, reducing the $y$-parameter, and leading to softer spectra, and thus the positive $\Gamma-L / L_{\text {edd }}$ correlation (e.g.. Janiuk \& Czerny 2000). The ADAF scenario has been relatively successful in explaining a variety of the LLAGN properties (Ho 2008), while the standard diskcorona model has been widely invoked to explain quasar emission. Our finding of a non-monotonic $\Gamma-L / L_{\text {edd }}$ relation seems to provide the first direct empirical link between these two different types of accretion in AGN.

The AGN-XRB physical analogy has been discussed rather extensively (see Maccarone, Fender \& Ho 2005), and is particularly supported by the two "fundamental planes" of the BH activity on all mass scales, the $L_{\text {radio }}-L_{X}-M_{b h}$ (Merloni, Heinz \& di Matteo 2003; Falcke. Körding \& Markoff 2004) and the $L_{\text {bol }}-M_{b h}-$ $T_{\text {break }}$ (McHardy et al. 2006). There was however not much evidence for existence of spectral states in massive black holes, similar to those of the stellar black holes. Our discovery of an anti-correlation between $\Gamma$ and $L / L_{\text {edd }}$, and thus the discovery of a turning point in the $\Gamma-L / L_{\text {edd }}$ relation for AGN, provides this evidence, which constitutes an important empirical constraint to the idea that these systems are really the analogs of each other, in spite of the vast difference of scales.

\section{CONCLUSIONS \& DISCUSSION}

This study of serendipitous Chandra nearby sources brings together for the first time a large homogeneous sample of active and inactive galaxy nuclei selected and classified based on their optical spectral properties. With a minimal selection bias, we characterize the X-ray properties of low luminosity AGN via measurements of the Xray spectral shape, fluxes, and luminosities. These measurements add important information and provide new constraints to the proposed $H I I \rightarrow S / T \rightarrow L \rightarrow P$ galaxy evolutionary sequence. Optical observations reveal that at least in statistical terms, along this sequence, (1) the host halo mass increases, (2) the environmental density increases, (3) both central BH mass and stellar mass increase, while (4) the rate of accretion onto the central
Bh decreases, (5) the stellar population ages, and (6) the material that could be used for accretion and/or starformation is less and less available. The X-ray data support these evolutionary trends and bring surprising new insights into the nature of the LLAGN phenomenon.

There are two main results of this analysis that we want to emphasize here:

- The $(H I I \rightarrow)$ Seyfert $\rightarrow$ Transition Object $\rightarrow$ LINER $\rightarrow$ Passive Galaxy sequence suggested by a large variety of optical measures is supported by $\mathrm{X}$-ray measurements. Both the spectral shape and the accretion power, as measured by $L_{X}$ and the Eddington ratio $L / L_{\text {edd }}$, with $L=L_{\text {bol }}=16 \times L_{X}$, show a clear trend toward softer, less X-ray luminous and less actively accreting sources from $S$ s to $T$ s, to $L \mathrm{~s}$, and, at the end, the Passive galaxies. The rather ambiguous (in some optical properties) succession of $S$ and $T$ phases is now significantly constrained by the X-ray activity to follow in a sense of decreasing accretion power.

- There is a rather strong anti-correlation between the shape of the X-ray spectral energy distribution, quantified via the power-law index $\Gamma$, and $L / L_{\text {edd }}$. This finding translates into a break in the $\Gamma-L / L_{\text {edd }}$ correlation exhibited by AGN of all powers, with spectral softening on either side of $L / L_{\text {edd }} \approx 0.01$. The transition point is identical to that where stellar mass $\mathrm{BH}$ accretors (XRBs) exhibit their turn in analogous $\Gamma-L / L_{\text {edd }}$ trends.

The distribution of points within the observed $\Gamma-$ $L / L_{\text {edd }}$ relation exhibited by both weak and powerful AGN might have other implications as well. The presence of some LLAGN below the $\Gamma \lesssim 1.5$ turning point, which are mostly Seyferts, suggests that obscuration might also play a role in shaping the observed $\Gamma-L / L_{\text {edd }}$ trends at low and high $z$. These particular objects' rather hard photon indices must be the indication of gas absorption of their $0.5-8 \mathrm{keV}$ spectra. Their Eddington ratios huddle near the $10^{-2}$ level, being generally weak when compared to the luminous quasars, and among the strongest LLAGN. Thus, the anti-correlation between $\Gamma$ and $L / L_{\text {edd }}$ shown by the nearby galaxy nuclei may well be interpreted as a relation between absorption and accretion rate, the objects accreting at higher rates being more obscured.

The probably naive extrapolation of this idea at higher $L / L_{\text {edd }}$ suggests then that more active AGN are also more absorbed. Consequently, these increasingly absorbed systems, that would be the type 2 ones [according to the AGN "unification" scenario that separates (the observed appearance of) AGN in terms of orientation relative to the line of sight] would be decreasingly likely to be included in the (current) optically selected AGN/quasar samples. This interpretation is certainly consistent with the general results of various quests for type 2 quasars: their X-ray spectra are harder than their type 1 counterparts (Zakamska et al. 2004; Ptak et al. 2006), and they are highly obscured (Zakamska et al. 2005). The type 2 quasars are definitely scarce compared with the type 1, and their fraction relative to that of the type 1 ones decreases with increasing luminosity (Reyes et al. 
2008). Thus, this may as well be the explanation for the dearth of type 2 quasars.

If the type 1-2 dichotomy and consequently the "unification" are only about the observing angle, the AGNgalaxy evolutionary sequence suggested by the properties of the different types of nearby galactic nuclei should be even stronger once inclination effects are removed, as we would have a clearer view of the central engine. On the other hand, the 1-2 type separation, and thus the unification, might be the result of evolution. Some simulations suggest that for the luminous quasars, the type 1 (unobscured) phase comes after a phase of "blowingout" circumnuclear matter, which might mean after the quasars were observable as type 2 . By adding type 1 LLAGN to investigations of the $\Gamma-L / L_{\text {edd }}$ connection we might be able to understand better the way the proposed evolutionary sequence does or does not challenge the unification scenario.

Also, the exact location of the turning point in the $\Gamma-L / L_{\text {edd }}$ relation remains to be better quantified in terms of both parameters. Of particular caution is combining the $L / L_{\text {edd }}$ measurements of LLAGN with those of quasars, mainly because the methods used in estimating their BH masses are not necessarily compatible. The $\mathrm{BH}$ masses of LLAGN are based on the $M_{b h}-\sigma_{*}$ relation, while the (usually high $z$ ) quasar BH masses are obtained from the widths of the optical broad emission lines via scaling relations; the scaling relations are calibrated on $M_{b h}-\sigma_{*}$, which, recent work suggests, does not necessarily hold at high $z$. Another way of refining the exact location of the break lies of course in better estimates of $\Gamma$, i.e., higher quality (higher signal-to-noise) $\mathrm{X}$-ray measurements, and/or larger samples. The latter alternative, in particular, seems to be feasible, as larger and larger samples of well characterized samples of optically selected AGN become available for cross-correlation with X-ray detections from, e.g., serendipitous surveys like ChaMP.

Future work (Constantin \& ChaMP 2009, in prep.) will explore the multi-wavelength properties of a large sample of AGN that brings together nearby LLAGN of type 2 with nearby quasars (type 1 AGN), thus attempting to reconcile both the type 1-2 dichotomy and the problem of mix-matching $\mathrm{BH}$ masses, along with providing larger statistics, and better means of quantifying extrinsic effects such as absorption and non-thermal processes (i.e., Comptonized emission from the accretion disk's corona) that enable improved constraints to the analogy with XRBs. We will also address in this work the biases that are potentially present in the previously claimed relationships between the between $L_{X}$ and optical emission line luminosities for LLAGN, together with the impact of using optical emission lines to estimate $L_{\mathrm{bol}}$, and thus to the overall shape of the $\Gamma-L / L_{\text {edd }}$ relation.

AC thanks Christy Tremonti for valuable discussions regarding the MPA/JHU catalog. Support for this work was provided by the National Aeronautics and Space Administration through Chandra Award Number AR78015A issued by the Chandra X-ray Observatory Center, which is operated by the Smithsonian Astrophysical Observatory for and on behalf of the National Aeronautics Space Administration under contract NAS8-03060.

\section{REFERENCES}

Aldcroft, T.L. 2006, Bulletin of the American Astronomical Society, 38, 376

Baldwin, J. A. Phillips, M. M, \& Terlevich, R., 1981, PASP, 93, 5 Barkhouse, W., et al., 2006, ApJ, 645, 955

Barth, A., \& Shields, C. J., 2000, PASP, 112, 753

Blandford, R.D., \& Begelman, M.C., 1999, MNRAS, 303, 1

Bohringer, H., et al. 2001, A\&A, 365, 181

Brinchmann, J., Charlot, S., Heckman, T. M., Kauffmann, G., Tremonti, C., \& White, S.D.M., 2004, astro-ph/0406220

Brinchmann, J., Charlot, S., White, S.D.M., Tremonti, C., Kauffmann, G., Heckman, T. M., \& Brinkmann, J., 2004, MNRAS, 351, 1151

Cappi, M., et al., 2006, A\&A, 446, 459

Cash, W. 1979, ApJ, 228, 939

Charlot, S., \& Fall, S. M., ApJ, 539, 718

Chiang, J., Reynolds, C. S., Blaes, O. M., Nowak, M. A., Murray, N., Madejski, G., Marshall, H. L., \& Magdziarz, P., 2000, ApJ, 528,292

Cid Fernandes, R., Gonzlez Delgado, R. M., Storchi-Bergmann, T., Martins, L.P., \& Schmitt, H., 2005, MNRAS, 356, 270

Colbert, E. J. M., Heckman, T. M., Ptak, A. F., Strickland, D. K.,\& Weaver, K. A., 2004, ApJ, 602, 231

Constantin, A., \& Vogeley, M.S., 2006, ApJ, 650, 727

Constantin, A., Hoyle, F., \& Vogeley, M.S., 2008, ApJ, 673, 715

Constantin, A., Shields, J.C., Ho, L.C., Barth, A., and Filippenko, A.V., 2009, in preparation.

Cowie, L. L.\& Barger, A. J., 2008, ApJ, 686, 72

Czerny, B., Nikolajuk, M., Raska, A., Dumont, A.-M., Loska, Z., Zycki, P. T., et al. 2003, A\&A, 412, 317

Dai, X., Chartas, G., Eracleous, M., Garmire, G.P., 2004, ApJ, 605,45

Dickey, J.M., \& Lockman, F.J., 1990, ARA\&A, 28, 215

Dudik, R. P., Satyapal, S., Gliozzi, M., \& Sambruna, R. M., 2005, ApJ, 620, 620, 113
Esin, A.A., McClintock, J.E., \& Narayan, R., 1997, ApJ, 489, 865 Eracleous, M., Shields, J. C., Chartas, G., \& Moran, E. C., 2002, ApJ, 565, 108

Falcke, H, Körding, E., \& Markoff, S., 2004, A\&A, 414, 895

Filho, M. E., Fraternali, F., Markoff, S., Nagar, N. M., Barthel, P. D., Ho, L. C., \& Yuan, F., 2004, A\&A, 418, 429

Filippenko, A. V., \& Terlevich, R., 1992, ApJ, 397, 79

Flohic, H.M.L.G., Eracleous, M., Chartas, G., Shields, J.C., \& Moran, E.C., 2006, ApJ, 647, 140

Forster, K. \& Halpern, J.P., 1996, ApJ, 468, 565

Georgantopoulos, I., Panessa, F., Akylas, A., Zezas, A., Cappi, M., \& Comastri, A., 2002, A\&A, 386, 60

George, I.M. \& Fabian A.C., 1991, MNRAS, 249, 352

George, I. M., Turner, T. J., Yaqoob, T., Netzer, H., Laor, A., Mushotzky, R. F., Nandra, K., \& Takahashi, T., 2000, ApJ, 531,52

Gierlinski, M. \& Done, C., 2004, MNRAS, 349, 7

Gonzalez-Martin, O., Masegosa, J., Marquez, I., Guerrero, M. A., \& Dultzin-Hacyan, D., 2006, A\&A, 460, 45

Green, P. J., et al., 2004, ApJS, 150, 43

Green, J.P., et al., 2009, ApJ, 690, 644

Greene, J.E., \& Ho, L.C., 2007, ApJ, 656, 84

Haardt, F. \& Maraschi, L., 1991, ApJ, 380, 51

Halderson, E.L., Moran, E.C., Filippenko, A.V., \& Ho, L.C., 2001, AJ, 122, 637

Heckman, T. M., Kauffmann, G., Brinchmann, J., Charlot, S., Tremonti, C., White, S. D. M., 2004, ApJ, 613, 109

Ho, L. C., Filippenko, A. V., \& Sargent, W. L. W., 1993, ApJ, 417,63

Ho, L. C., Filippenko, A. V., \& Sargent, W. L. W., 1997a, ApJS, 112,315

Ho, L. C., Filippenko, A. V., \& Sargent, W. L. W., 1997b, ApJ, 487,568 
Ho, L. C., Feigelson, E., D., Townsley, L. K., Sambruna, R. M., Garmire, G. P., Brandt, W. N., Filippenko, A. V., Griffiths, R. E., Ptak, A. F., \& Sargent, W. L. W. 2001, ApJ, 549, L51

Ho, L. C., Filippenko, A. V., \& Sargent, W. L. W., 2003, ApJ, 583,159

Ho, L. C., 2008, ARA\&A, 46, 475

Hopkins, P.F., \& Hernquist, L., 2006, ApJ, 166, 1

Hopkins, P.F., \& Hernquist, L., 2009, ApJ, in press (arXiv0809.3789)

Ishisaki, Y., et al., 1996, PASJ, 48, 237

Iyomoto, N., Makishima, K., Matsushita, K., Fukazawa, Y., Tashiro, M., \& Ohashi, T., 1998, ApJ, 503, 168

Iyomoto, N., Fukazawa, Y., Nakai, N., \& Ishihara, Y., 2001, ApJ, 561,69

Janiuk, A., \& Czerny, B., 2000, NewA, 5, 7

Kalemci, E., Tomsick, J. A., Buxton, M. M., Rothschild, R. E., Pottschmidt, K., Corbel, S., Brocksopp, C., \& Kaaret, P., ApJ, 622,508

Kauffmann, G., et al., 2003, MNRAS, 341, 33

Kauffmann, G., et al., 2003, MNRAS, 346, 105

Kauffmann, G., et al., 2004, MNRAS, 353, 713

Kauffmann, G., \& Heckman, T.M., 2009, MNRAS, submitted (arXiv0812.1224)

Kelly, B. C., Bechtold, J., Trump, J. R., Vestergaard, M., \& Siemiginowska, A., 2008, ApJ, 176, 355

Kewley, L., Dopita, M. A., Sutherland, R. S., Heisler, C. A., \& Trevena, J., 2001, ApJ, 556, 121

Kewley, L.J., Groves, B., Kauffmann, G., \& Heckman, T., 2006, MNRAS, 372, 961

Kim, D.-W., Fabbiano, G., \& Trinchieri, G., 1992, ApJ, 393, 134

Kim, D.-W., Fabbiano, G., \& Trinchieri, G., 1992, ApJS, 80, 645

Kim, D.-W. \& Fabbiano, G., 2003, ApJ, 586, 826

Kollmeier, J.A., et al., 2006, ApJ, 648, 128

Kubota, A., \& Makishima,K., 2004, ApJ, 601, 428

Lehmer, B., et al. 2005, ApJ, 129, 1

Lightman, A.P. \& White, T.R., 1988, ApJ, 335, 57

Lu, Y. \& Yu, Q, 1999, ApJ, 526, 5

Lu, J.-F., Li, S.-L., Gu, W.-M., 2004, MNRAS, 352, 147

Maccarone, T.J., Fender, R.P., \& Ho, L.C., 2005, From X-ray Binaries to Quasars: Black Hole Accretion on All Mass Scales Dordrecht:Luwer,

McHardy, I. M., Koerding, E., Knigge, C., Uttley, P., Fender, R. P., 2006, Nature, 444, 730

McLure, R.J., \& Dunlop,J.S., 2004, MNRAS, 352, 1390

Merloni, A., Heinz, S., di Matteo, T., 2003, MNRAS, 345, 1057

Miniutti, G., Ponti, G., Greene, J. E., Ho, L. C., Fabian, A. C., Iwasawa, K., 2008, MNRAS, 394, 443

Nandra, K. \& Pounds, K.A., 1994, MNRAS, 268, 405

Narayan, R., \& Yi, I., 1994, ApJ, 428, 13

Narayan, R., Igumenshchev, \& I. V., Abramowicz, M. A., 2000, ApJ, 539, 798

Narayan, R., \& McClintock, J.E., 2008, New Astronomy Reviews, 51,733

Nayakshin, S., 2000, ApJ, 540, 37

Netzer, H., Lira, P., Trakhtenbrot, B., Shemmer, Ohad, \& Cury, I., 2007, ApJ, 671, 1256

Osterbrock, D. E., 1989, Astrophysics of Gaseus Nebulae and Active Galactic Nuclei, University Science Books

Page, M. J., Soria, R., Zane, S., Wu, K., \& Starling, R. L. C., 2004, A\&A, 422, 77

Panessa, F., Bassani, L., Cappi, M., Dadina, M., Barcons, X., Carrera, F. J., Ho, L. C., \& Iwasawa, K., L., 2006, A\&A, 455,173

Parejko, J. K., Constantin, A., Vogeley, M. S., \& Hoyle, F., 2008, AJ, 135, 10

Pellegrini, S., Cappi, M., Bassani, L., Malaguti, G., Palumbo, G. G. C., \& Persic, M., 2000a, A\&A, 353, 447

Pellegrini, S., Cappi, M., Bassani, L., della Ceca, R., \& Palumbo, G. G. C., 2000b, A\&A, 360, 878

Pellegrini, S., Fabbiano, G., Fiore, F., Trinchieri, G., \& Antonelli, A., 2002, A\&A, 383, 1

Pellegrini, S., Baldi, A., Fabbiano, G., \& Kim, D.-W., 2003, ApJ, 597, 175

Pellegrini, S., 2005, ApJ, 624, 155

Petrucci, P. O., Haardt, F., Maraschi, L., Grandi, P., Matt, G., Nicastro, F., Piro, L., Perola, G. C., \& De Rosa, A., 2000, ApJ, 540,131
Porquet, D., Reeves, J. N., O’Brien, P., \& Brinkmann, W., A\&A, 422, 85

Poutanen, J., Krolik, J. H., Ryde, F., 1997, MNRAS, 292, 21

Proga, D., 2005, ApJ, 630, 9

Ptak, A., Yaqoob, T., Serlemitsos, P. J., Kunieda, H., \& Terashima, Y., 1996, ApJ, 459, 542

Ptak, A., Serlemitsos, P., Yaqoob, T., \& Mushotzky, 1999, ApJS, 120,179

Ptak, A., Terashima, Y., Ho, L. C., Quataert, E., 2004, ApJ, 606 , 173

Ptak, A., Zakamska, N. L., Strauss, M. A., Krolik, J. H., Heckman, T. M., Schneider, D. P., \& Brinkmann, J., 2006, ApJ, 637, 147

Reddy, N.A., \& Steidel, C.C., 2004, ApJ, 603, 13

Reyes, R., Zakamska, N. L., Strauss, M. A., Green, J., Krolik, J. H., Shen, Y., Richards, G. T., Anderson, S. F., Schneider, D. P., 2008, AJ, 136, 2373

Roberts, T. P., Warwick, R. S., 2000, MNRAS, 315, 98

Roberts, T. P., Schurch, N. J., Warwick, R. S., 2001, MNRAS, 324,737

Satyapal, S., Sambruna, R. M., \& Dudik, R. P., 2004, A\&A, 414, 825

Satyapal, S., Dudik, R. P., O'Halloran, B., Gliozzi, M., 2005, ApJ, 633, 86

Shakura, N. I., \& Syunyaev, R. A., 1973, A\&A, 24, 337

Shapiro, S. L., Lightman, A. P., \& Eardley, D. M., 1976, ApJ, 204,187

Shemmer, O., Brandt, W. N., Netzer, H., Maiolino, R., \& Kaspi, S., 2008, ApJ, 682, 81

Shen, Y., Greene, J. E., Strauss, M. A., Richards, G.T., Schneider, D.P., 2008, ApJ, 680, 169

Shields, J. C., 1992, ApJ, 399, 27L

Sobolewska, M.A., Siemiginowska, A., \& ycki, P.T., 2004, ApJ, 617, 102

Soria, R., Fabbiano, G., Graham, A.W., Baldi, A., Elvis, M., Jerjen, H., Pellegrini, S., \& Siemiginowska, A., 2006, ApJ, 640, 126

Starling, R. L. C., Page, M. J., Branduardi-Raymont, G., Breeveld, A. A., Soria, R., \& Wu, K., 2005, MNRAS, 356, 727

Stasiska, G., Asari, N.V., Fernandes, R.C., Gomes, J.M., Schlickmann, M., Mateus, A., Schoenell, W., \& Sodr, L., Jr., 2008, MNRAS, 391, 29

Taniguchi, Y., Shioya, Y., \& Murayama, T., 2000, AJ, 120, 1265

Terashima, Y., et al., 1998

Terashima, Y., Ho, L.C., Ptak, A.F., 2000a, ApJ, 539, 161

Terashima, Y., Ho, L.C., Ptak, A.F., Mushotzky, R. F., Serlemitsos, P. J., Yaqoob, T., Kunieda, H., 2000b, ApJ, 533, 729

Terashima, Y., Iyomoto, N., Ho, Luis C., Ptak, A.F., 2002, ApJS, 139,1

Terashima, Y., \& Wilson, A. S., 2003, ApJ, 583,145

Tremaine, S., et al., 2002, ApJ, 574, 740

Tremonti, C.A., et al., 2004, ApJ, 613, 898

Veilleux, S., \& Osterbrock, D. E., 1987, ApJS, 63, 295

Vignali, C., Brandt, W. N., Schneider, D. P., Kaspi, S., 2005, AJ, 129,2519

Vaughan, S., Edelson, R., Warwick, R. S., Malkan, M. A., \& Goad, M. R., 2001, MNRAS, 327, 673

Wang, J.-M., Watarai, K.-Y., \& Mineshige, S., 2004, ApJ, 607, 107

Williams, R. J., Mathur, S., \& Pogge, R.W., 2004, ApJ, 610, 737

Winter, L. M., Mushotzky, R. F., Reynolds, C. S., Tueller, J., 2009, ApJ, 690, 1322

Wu, Q., \& Gu, M., 2008, ApJ, 682, 212

Yaqoob, T., Edelson, R., Weaver, K. A., Warwick, R. S., Mushotzky, R. F., Serlemitsos, P. J., Holt, S. S., 1995, ApJ, 453,81

Yamaoka, K., Uzawa, M., Arai, M., Yamazaki, T., \& Yoshida, A, 2005, ChJAS, 5, 273

Yu, Q., \& Tremaine, S., 2002, MNRAS, 335, 965

Yuan, F. \& Narayan, R., 2004, ApJ, 612, 724

Yuan, F., Taam, R. E., Misra, R., Wu, X.-B., \& Xue, Y., 2007, ApJ, 658, 282

Zakamska, N. L., Strauss, M. A., Heckman, T. M.; Ivezi, ., Krolik, J. H., 2004, AJ, 128, 1002 
Zakamska, N. L., Schmidt, G. D., Smith, P. S., Strauss, M. A., Krolik, J. H., Hall, P. B., Richards, G. T., Schneider, D. P., Brinkmann, J., Szokoly, G. P., 2005, AJ, 129, 1212

Zdziarski, A. A., Lubinski, P., Smith, David A., 1999, MNRAS, 303, 11
Zhang, X.-G., Dultzin, D., \& Wang, T.-G., 2008, MNRAS, 385, 1087 
TABLE 1

X-ray measurements of ChaMP-SDSS Galaxies

\begin{tabular}{|c|c|c|c|c|c|c|c|c|c|c|c|c|c|c|}
\hline $\begin{array}{l}\text { ObjID } \\
(1)\end{array}$ & $\begin{array}{l}\mathrm{ra} \\
(2)\end{array}$ & $\begin{array}{l}\text { dec } \\
(3)\end{array}$ & $\begin{array}{c}z \\
(4)\end{array}$ & $\begin{array}{c}\text { srcid } \\
(5)\end{array}$ & $\begin{array}{c}\text { OAA } \\
(6)\end{array}$ & $\begin{array}{c}\text { counts } \\
(7)\end{array}$ & $\begin{array}{c}\text { Exp. } \\
(8)\end{array}$ & $\begin{array}{l}\text { Targ. } \\
(9)\end{array}$ & $\begin{array}{c}N_{H}^{G a l} \\
(10)\end{array}$ & $\begin{array}{c}N_{H}^{I n t r .} \\
(11)\end{array}$ & $\begin{array}{c}\Gamma \\
(12)\end{array}$ & $\begin{array}{c}f_{x} \\
(13)\end{array}$ & $\begin{array}{l}L_{X} \\
(14)\end{array}$ & $\begin{array}{l}\text { Opt. Class } \\
\text { (15) }\end{array}$ \\
\hline 587722982300254964 & 233.296661 & -0.756684 & 0.151 & CXOMP J153311.1-004524 & 6.8 & 27.6 & 4.9 & $\ldots$ & 6.39 & $6.9_{-2.4}^{+3.6}$ & $-0.7_{-0.6}^{+0.5}$ & 218.41 & 42.94 & $\mathrm{~S}$ \\
\hline 587722983362134277 & 206.118027 & 0.029700 & 0.135 & CXOMP J134428.3+000146 & 2.4 & 5.7 & 8.7 & $\ldots$ & 1.93 & $¡ 0.8$ & $2.1_{-1.1}^{+1.3}$ & 5.14 & 41.06 & $\mathrm{P}$ \\
\hline 587722984431026387 & 195.126404 & 1.046143 & 0.067 & CXOMP J130030.3+010246 & 8.4 & 16.6 & 1.5 & $\ldots$ & 1.63 & $¡ 1.3$ & $1.3_{-0.6}^{+0.1}$ & 152.65 & 42.05 & $\mathrm{~T}$ \\
\hline 587725041163370794 & 174.768066 & -1.980904 & 0.342 & CXOMP J113904.3-015851 & 3.5 & 2.5 & 14.0 & $\ldots$ & 2.56 & $¡ 0.4$ & $4.3_{-2.5}^{+5.0}$ & 1.03 & 40.50 & $\mathrm{P}$ \\
\hline 587725470127161548 & 118.935455 & 41.204029 & 0.074 & CXOMP J075544.5+411214 & 10.1 & 33.9 & 7.2 & $\ldots$ & 4.62 & $¡ 0.0$ & $3.0_{-0.6}^{+0.7}$ & 50.35 & 41.14 & $\mathrm{P}$ \\
\hline 587725550133117038 & 155.188263 & 63.196468 & 0.206 & CXOMP J102045.1+631147 & 4.2 & 39.8 & 6.0 & $\ldots$ & 1.03 & $¡ 0.0$ & $2.4_{-0.4}^{+0.0}$ & 51.62 & 42.38 & $\mathrm{~T}$ \\
\hline 587725550136983727 & 174.983627 & 66.098259 & 0.376 & CXOMP J113956.0+660553 & 5.6 & 15.0 & 114.6 & $\ldots$ & 1.17 & ¡0.3 & $2.8_{-0.8}^{+1.4}$ & 0.99 & 41.19 & $\mathrm{P}$ \\
\hline 587725551735996593 & 127.912956 & 52.701363 & 0.058 & CXOMP J083139.1+524204 & 3.7 & 23.5 & 6.8 & $\ldots$ & 3.91 & $¡ 1.4$ & $-0.4_{-0.6}^{+0.8}$ & 87.79 & 41.77 & $\mathrm{~S}$ \\
\hline 587725591458414903 & 264.505035 & 58.503334 & 0.330 & CXOMP J173801.2+583012 & 3.3 & 138.8 & 4.5 & $\ldots$ & 3.56 & $¡ 0.1$ & $1.8_{-0.4}^{+0.0}$ & 192.16 & 43.55 & $\mathrm{~T}$ \\
\hline 587725980689301524 & 128.116867 & 52.605656 & 0.016 & CXOMP J083228.0+523620 & 11.6 & 41.7 & 9.0 & $\ldots$ & 3.84 & $¡ 0.0$ & $2.9_{-0.5}^{+0.4}$ & 45.71 & 39.77 & H II \\
\hline 587726015607275687 & 183.399002 & 2.810350 & 0.132 & CXOMP J121335.7+024837 & 2.2 & 11.4 & 18.0 & $\ldots$ & 1.75 & $¡ 0.8$ & $1.6_{-0.8}^{+0.8}$ & 7.37 & 41.31 & $\mathrm{~L}$ \\
\hline 587726015607275717 & 183.441772 & 2.811480 & 0.073 & CXOMP J121346.0+024841 & 4.2 & 10.4 & 18.6 & $\ldots$ & 1.75 & ¡0.6 & $2.0_{-0.9}^{+1.0}$ & 13.05 & 40.90 & $\mathrm{~T}$ \\
\hline 587726031729459313 & 220.677521 & 1.319711 & 0.033 & CXOMP J144242.6+011910 & 8.1 & 119.7 & 10.9 & $\ldots$ & 3.35 & $¡ 0.2$ & $1.6_{-0.5}^{+0.9}$ & 106.12 & 41.22 & $\mathrm{~L}$ \\
\hline 587726033305338033 & 141.248947 & 2.241570 & 0.148 & CXOMP J092459.7+021429 & 4.4 & 7.3 & 17.1 & $\ldots$ & 3.73 & ¡3.2 & $0.5_{-1.0}^{+0.9}$ & 8.14 & 41.53 & no-class \\
\hline 587726100949238048 & 219.718658 & 3.716406 & 0.291 & CXOMP J143852.4+034259 & 9.0 & 22.6 & 54.6 & $\ldots$ & 2.63 & $¡ 0.1$ & $3.7_{-0.9}^{+1.0}$ & 5.71 & 41.28 & $\mathrm{P}$ \\
\hline 587727179536138389 & 30.007231 & -8.927990 & 0.052 & CXOMP J020001.7-085540 & 4.8 & 29.9 & 34.2 & $\ldots$ & 2.09 & $¡ 0.2$ & $2.0_{-0.5}^{+0.9}$ & 8.64 & 40.43 & S \\
\hline 587727213347209620 & 322.206177 & -7.787057 & 0.070 & CXOMP J212849.4-074713 & 10.1 & 12.7 & 19.5 & $\ldots$ & 4.88 & $21.6_{-9.1}^{+51.2}$ & $-1.3_{-2.5}^{+1.4}$ & 28.58 & 41.43 & $\mathrm{H}$ II \\
\hline 587727223009510128 & 325.644348 & 12.505494 & 0.276 & CXOMP J214234.6+123019 & 2.6 & 5.0 & 14.0 & $\ldots$ & 6.69 & $¡ 1.3$ & $2.0_{-1.1}^{+1.3}$ & 2.70 & 41.49 & $\mathrm{P}$ \\
\hline 587727227305394267 & 10.728508 & -9.230717 & 0.076 & CXOMP J004254.8-091350 & 7.3 & 13.4 & 9.6 & $\ldots$ & 3.53 & $¡ 0.1$ & $4.0_{-1.0}^{+1.1}$ & 19.63 & 40.24 & $\mathrm{~L}$ \\
\hline 587727227305394310 & 10.752554 & -9.229577 & 0.076 & CXOMP J004300.6-091346 & 7.7 & 63.5 & 9.6 & $\ldots$ & 3.54 & $¡ 0.1$ & $2.5_{-0.4}^{+0.4}$ & 69.02 & 41.50 & $\mathrm{~L}$ \\
\hline 587727884161581244 & 29.994183 & -8.826961 & 0.392 & CXOMP J015958.6-084937 & 2.1 & 19.3 & 34.8 & $\ldots$ & 2.11 & $¡ 2.1$ & $1.3_{-0.6}^{+0.4}$ & 7.32 & 42.35 & $\mathrm{H}$ II \\
\hline 587727884161581256 & 29.955580 & -8.833209 & 0.405 & CXOMP J015949.3-084959 & 1.6 & 917.4 & 34.8 & I & 2.10 & $¡ 0.2$ & $1.9_{-0.1}^{+0.0}$ & 254.49 & 43.88 & no-class \\
\hline 587727942420988082 & 144.690018 & 0.990713 & 0.171 & CXOMP J093845.6+005926 & 1.7 & 16.9 & 1.2 & $\ldots$ & 4.22 & $¡ 0.4$ & $1.4_{-0.6}^{+0.1}$ & 114.19 & 42.76 & $\mathrm{~T}$ \\
\hline 587728307491897590 & 168.966110 & 1.498703 & 0.352 & CXOMP J111551.8+012955 & 2.1 & 286.1 & 15.1 & I & 4.37 & $¡ 0.2$ & $2.1_{-0.2}^{+0.0}$ & 168.98 & 43.52 & $\mathrm{~T}$ \\
\hline 587728905564258619 & 120.236748 & 36.056530 & 0.287 & CXOMP J080056.8+360323 & 1.3 & 496.6 & 35.4 & I & 4.95 & $0.1_{-0.1}^{+0.1}$ & $2.0_{-0.3}^{+0.3}$ & 90.87 & 43.10 & no-class \\
\hline 587729157893456002 & 196.494354 & 3.956814 & 0.023 & CXOMP J130558.6+035724 & 3.0 & 55.1 & 112.0 & $\ldots$ & 2.08 & $\jmath^{-0.1}$ & $1.3_{-0.3}^{+0.3}$ & 4.26 & 39.56 & $\mathrm{~T}$ \\
\hline 587729157893456055 & 196.563110 & 3.931599 & 0.110 & CXOMP J130615.1+035553 & 1.6 & 16.8 & 112.0 & $\ldots$ & 2.08 & $¡ 0.1$ & $2.5_{-0.8}^{+1.0}$ & 0.76 & 39.91 & $\mathrm{~T}$ \\
\hline 587729158440419656 & 219.591049 & 3.670265 & 0.224 & CXOMP J143821.8+034012 & 3.6 & 37.0 & 56.2 & I & 2.61 & $¡ 0.4$ & $1.9_{-0.5}^{+0.5}$ & 7.36 & 41.74 & no-class \\
\hline 587729158440419712 & 219.609650 & 3.649648 & 0.235 & CXOMP J143826.3+033858 & 2.3 & 19.6 & 56.2 & $\ldots$ & 2.61 & $¡ 0.1$ & $3.8_{-0.9}^{+1.0}$ & 3.42 & 40.79 & $\mathrm{P}$ \\
\hline 587729388215337158 & 139.517227 & 51.687130 & 0.186 & CXOMP J091804.1+514113 & 6.2 & 23.4 & 18.7 & $\ldots$ & 1.48 & $12.1_{-5.3}^{+7.3}$ & $-0.6_{-0.7}^{+0.9}$ & 43.52 & 42.41 & $\mathrm{~T}$ \\
\hline 587729751132667997 & 247.652924 & 40.131489 & 0.077 & CXOMP J163036.7+400753 & 4.2 & 10.8 & 26.0 & $\ldots$ & 0.88 & ¡0.1 & $3.4_{-1.0}^{+1.2}$ & 3.53 & 39.86 & $\mathrm{~T}$ \\
\hline 587729751132668135 & 247.629547 & 40.156597 & 0.077 & CXOMP J163031.0+400923 & 2.7 & 20.6 & 24.8 & $\ldots$ & 0.88 & $¡ 0.1$ & $3.4_{-0.9}^{+1.0}$ & 3.56 & 39.83 & $\mathrm{~T}$ \\
\hline 587729752213815608 & 260.041504 & 26.625124 & 0.159 & CXOMP J172009.9+263730 & 2.2 & 566.4 & 24.0 & I & 3.89 & ¡0.2 & $2.1_{-0.2}^{+0.3}$ & 209.66 & 42.84 & $\mathrm{~T}$ \\
\hline 587729753280741824 & 250.473267 & 40.029160 & 0.466 & CXOMP J164153.5+400144 & 2.5 & 23.4 & 45.3 & I & 1.02 & $¡ 0.3$ & $2.3_{-0.6}^{+0.8}$ & 4.84 & 42.24 & no-class \\
\hline 587730816826671317 & 340.846375 & -9.518315 & 0.144 & CXOMP J224323.1-093105 & 6.4 & 32.9 & 18.2 & $\ldots$ & 4.33 & $3.0_{-1.1}^{+1.6}$ & $0.1_{-0.5}^{+0.6}$ & 45.66 & 42.25 & $\mathrm{~S}$ \\
\hline 587731185129816192 & 359.235931 & -0.986925 & 0.032 & CXOMP J235656.6-005912 & 1.2 & 9.2 & 17.9 & $\ldots$ & 3.52 & $¡ 0.3$ & $2.1_{-0.9}^{+1.3}$ & 4.77 & 39.73 & $\mathrm{~L}$ \\
\hline 587731186729550057 & 334.344727 & 0.351939 & 0.095 & CXOMP J221722.7+002106 & 6.1 & 14.6 & 75.4 & $\ldots$ & 4.61 & $¡ 0.5$ & $1.5_{-0.7}^{+0.8}$ & 2.23 & 40.51 & $\mathrm{~T}$ \\
\hline 587731187277430877 & 359.432159 & 0.654831 & 0.023 & CXOMP J235743.7+003917 & 7.4 & 147.9 & 11.9 & $\ldots$ & 3.32 & $1.7_{-0.5}^{+0.6}$ & $0.9_{-0.2}^{+0.2}$ & 438.43 & 41.62 & S \\
\hline 587731187277430877 & 359.431946 & 0.655582 & 0.023 & CXOMP J235743.6+003920 & 7.4 & 142.7 & 11.9 & $\ldots$ & 3.32 & $1.9_{-0.6}^{+0.7}$ & $0.5_{-0.3}^{+0.3}$ & 525.45 & 41.73 & S \\
\hline 587731187813253146 & 357.074768 & 1.104287 & 0.092 & CXOMP J234817.9+010615 & 7.7 & 36.5 & 48.7 & $\ldots$ & 3.96 & $¡ 0.04$ & $3.4_{-0.6}^{+0.3}$ & 6.36 & 40.25 & $\mathrm{P}$ \\
\hline 587731511532453955 & 19.723360 & -1.002003 & 0.045 & CXOMP J011853.6-010007 & 1.7 & 217.6 & 38.3 & I & 3.67 & $0.7_{-0.3}^{+0.4}$ & $5.7_{-1.5}^{+2.0}$ & 28.49 & 40.62 & no-class \\
\hline 587731512613404741 & 36.155399 & -0.047232 & 0.127 & CXOMP J022437.2-000250 & 0.8 & 12.0 & 86.7 & $\ldots$ & 2.85 & $¡ 0.6$ & $1.7_{-0.7}^{+0.9}$ & 1.21 & 40.47 & $\mathrm{~T}$ \\
\hline
\end{tabular}


TABLE $1-$ Continued

\begin{tabular}{|c|c|c|c|c|c|c|c|c|c|c|c|c|c|c|}
\hline $\begin{array}{l}\text { ObjID } \\
(1)\end{array}$ & $\begin{array}{l}\mathrm{ra} \\
(2)\end{array}$ & $\begin{array}{l}\text { dec } \\
(3)\end{array}$ & $\begin{array}{c}z \\
(4)\end{array}$ & $\begin{array}{c}\text { srcid } \\
(5)\end{array}$ & $\begin{array}{c}\text { OAA } \\
(6)\end{array}$ & $\begin{array}{l}\text { counts } \\
(7)\end{array}$ & $\begin{array}{c}\text { Exp. } \\
(8)\end{array}$ & $\begin{array}{c}\text { Targ. } \\
(9)\end{array}$ & $\begin{array}{c}N_{H}^{G a l} \\
(10)\end{array}$ & $\begin{array}{c}N_{H}^{\text {Intr. }} \\
(11)\end{array}$ & $\begin{array}{c}\Gamma \\
(12)\end{array}$ & $\begin{array}{c}f_{x} \\
(13)\end{array}$ & $\begin{array}{c}L_{X} \\
(14)\end{array}$ & $\begin{array}{l}\text { Opt. Class } \\
\text { (15) }\end{array}$ \\
\hline 587731513148571800 & 32.354515 & 0.399077 & 0.061 & CXOMP J020925.0+002356 & 9.9 & 29.2 & 2.6 & $\ldots$ & 2.79 & ¡0.4 & $1.6_{-0.5}^{+0.5}$ & 138.82 & 41.88 & $\mathrm{~T}$ \\
\hline 587731868557377700 & 168.673538 & 53.250507 & 0.106 & CXOMP J111441.6+531501 & 4.8 & 23.4 & 16.8 & $\ldots$ & 0.92 & $7.2_{-2.7}^{+5.3}$ & $-0.6_{-0.6}^{+0.6}$ & 43.22 & 41.96 & $\mathrm{~L}$ \\
\hline 587731873388101721 & 123.742249 & 36.890556 & 0.108 & CXOMP J081458.1+365326 & 8.2 & 10.6 & 9.3 & $\ldots$ & 5.02 & $¡ 0.2$ & $2.4_{-1.1}^{+1.5}$ & 10.99 & 41.09 & $\mathrm{~S}$ \\
\hline 587731886272741666 & 123.901619 & 36.765156 & 0.174 & CXOMP J081536.3+364554 & 6.1 & 9.3 & 8.8 & $\ldots$ & 5.00 & ¡0.3 & $3.1_{-1.0}^{+1.3}$ & 10.98 & 41.30 & $\mathrm{P}$ \\
\hline 587732050021318723 & 148.205582 & 51.884888 & 0.215 & CXOMP J095249.3+515305 & 1.1 & 283.2 & 24.4 & I & 0.88 & ¡0.5 & $2.6_{-0.5}^{+0.0}$ & 59.21 & 42.60 & $\mathrm{~L}$ \\
\hline 587732135913521261 & 150.952209 & 47.631630 & 0.051 & CXOMP J100348.5+473753 & 0.9 & 13.5 & 13.5 & $\ldots$ & 0.93 & $¡ 0.1$ & $2.7_{-0.8}^{+1.0}$ & 4.57 & 39.90 & $\mathrm{~T}$ \\
\hline 587732469851553889 & 131.364151 & 34.419083 & 0.025 & CXOMP J084527.3+342508 & 4.3 & 4.6 & 4.0 & $\ldots$ & 3.42 & $¡ 0.3$ & $2.6_{-1.4}^{+2.2}$ & 7.92 & 39.56 & $\mathrm{~T}$ \\
\hline 587732482200371401 & 145.504807 & 41.440948 & 0.243 & CXOMP J094201.1+412627 & 6.3 & 11.4 & 7.5 & $\ldots$ & 0.95 & ¡0.1 & $4.0_{-1.2}^{+1.5}$ & 6.62 & 41.01 & $\mathrm{P}$ \\
\hline 587732484351983843 & 156.306671 & 47.115517 & 0.060 & CXOMP J102513.6+470655 & 10.0 & 5.8 & 2.1 & $\ldots$ & 1.22 & ¡1.0 & $1.9_{-1.1}^{+1.3}$ & 31.99 & 41.16 & $\mathrm{~T}$ \\
\hline 587733397572747445 & 244.418823 & 35.004284 & 0.029 & CXOMP J161740.5+350015 & 0.6 & 151.7 & 18.3 & $\mathrm{I}$ & 1.47 & ¡0.02 & $2.7_{-0.4}^{+0.4}$ & 35.71 & 40.29 & $\mathrm{~L}$ \\
\hline 587733603191161025 & 240.295242 & 43.194328 & 0.071 & CXOMP J160110.8+431139 & 7.5 & 255.5 & 26.7 & $\ldots$ & 1.31 & $¡ 5.8$ & $0.9_{-0.8}^{+0.9}$ & 294.80 & 42.47 & $\mathrm{~S}$ \\
\hline 587733604801708195 & 241.492157 & 44.055454 & 0.044 & CXOMP J160558.1+440319 & 3.6 & 1324.3 & 4.6 & $\ldots$ & 1.15 & ¡0.01 & $1.9_{-0.0}^{+0.0}$ & 1619.01 & 42.56 & S \\
\hline 587734304342212857 & 336.311615 & -0.364847 & 0.142 & CXOMP J222514.7-002153 & 7.5 & 6.2 & 3.1 & $\ldots$ & 5.12 & ¡0.5 & $2.5_{-1.2}^{+1.5}$ & 29.36 & 41.76 & $\mathrm{~T}$ \\
\hline 587735348561051857 & 151.805237 & 12.787229 & 0.248 & CXOMP J100713.2+124714 & 4.0 & 18.0 & 36.3 & $\ldots$ & 3.68 & $¡ 0.1$ & $4.2_{-1.0}^{+1.1}$ & 2.60 & 40.55 & no-class \\
\hline 587735661546504321 & 143.357269 & 34.048050 & 0.027 & CXOMP J093325.7+340252 & 2.5 & 33.6 & 33.6 & $\mathrm{I}$ & 1.47 & ¡0.03 & $3.6_{-0.6}^{+0.6}$ & 9.81 & 39.24 & $\mathrm{~L}$ \\
\hline 587735661546504414 & 143.272659 & 34.062729 & 0.277 & CXOMP J093305.4+340345 & 6.8 & 23.1 & 33.6 & $\ldots$ & 1.47 & $¡ 0.4$ & $1.5_{-0.6}^{+0.6}$ & 8.69 & 42.08 & $\mathrm{~T}$ \\
\hline 587735662089666625 & 159.883911 & 39.836349 & 0.068 & CXOMP J103932.1+395010 & 2.7 & 10.7 & 5.0 & $\ldots$ & 1.43 & $¡ 0.9$ & $0.8_{-0.7}^{+0.7}$ & 25.60 & 41.33 & $\mathrm{~T}$ \\
\hline 587735666377883763 & 206.114655 & 56.024902 & 0.070 & CXOMP J134427.5+560129 & 7.5 & 45.1 & 43.5 & $\ldots$ & 1.09 & $0.9_{-0.4}^{+0.6}$ & $0.8_{-0.4}^{+0.4}$ & 16.90 & 41.19 & no-class \\
\hline 587735666377883859 & 206.093246 & 55.951073 & 0.038 & CXOMP J134422.3+555703 & 3.9 & 7.4 & 42.7 & $\ldots$ & 1.09 & $i_{0.2}^{-0.4}$ & $3.2_{-1.1}^{+1.3}$ & 1.22 & 38.85 & $\mathrm{~T}$ \\
\hline 587735695377432678 & 214.062256 & 53.146236 & 0.114 & CXOMP J141614.9+530846 & 5.7 & 8.1 & 60.5 & $\ldots$ & 1.27 & $¡ 22.5$ & $-0.1_{-1.1}^{+0.9}$ & 3.95 & 40.99 & no-class \\
\hline 587735696440623133 & 171.764420 & 56.902294 & 0.055 & CXOMP J112703.4+565408 & 3.3 & 7.8 & 38.3 & $\ldots$ & 0.92 & $¡ 0.5$ & $1.5_{-1.0}^{+1.3}$ & 2.26 & 40.02 & no-class \\
\hline 587735696440623158 & 171.882370 & 56.876919 & 0.005 & CXOMP J112731.7+565236 & 0.8 & 45.3 & 39.3 & $\ldots$ & 0.90 & ¡0.4 & $0.6_{-0.4}^{+0.4}$ & 16.00 & 38.99 & $\mathrm{H}$ II \\
\hline 587735696440623212 & 171.780807 & 56.828369 & 0.175 & CXOMP J112707.3+564942 & 3.8 & 12.0 & 38.3 & $\ldots$ & 0.91 & ¡0.1 & $3.8_{-1.1}^{+1.6}$ & 2.48 & 40.32 & $\mathrm{P}$ \\
\hline 587735696448880650 & 206.089798 & 55.856201 & 0.037 & CXOMP J134421.5+555122 & 4.1 & 9.6 & 42.7 & $\ldots$ & 1.08 & $¡ 0.2$ & $2.2_{-0.9}^{+1.2}$ & 1.70 & 39.35 & no-class \\
\hline 587735696451371138 & 215.187088 & 53.653847 & 0.117 & CXOMP J142044.9+533913 & 6.4 & 126.2 & 4.9 & $\ldots$ & 1.19 & ¡0.1 & $1.9_{-0.3}^{+0.4}$ & 167.86 & 42.48 & $\mathrm{~S}$ \\
\hline 587735696979656747 & 180.807953 & 57.890541 & 0.034 & CXOMP J120313.9+575325 & 5.9 & 93.9 & 57.8 & $\ldots$ & 1.43 & ¡0.2 & $1.9_{-0.2}^{+0.3}$ & 19.12 & 40.43 & H II \\
\hline 587735744228753459 & 245.066696 & 29.488642 & 0.061 & CXOMP J162016.0+292919 & 0.6 & 10.7 & 32.2 & $\ldots$ & 2.74 & ¡12.6 & $0.1_{-0.8}^{+0.7}$ & 6.82 & 40.69 & no-class \\
\hline 587736584961196166 & 205.136429 & 40.293945 & 0.171 & CXOMP J134032.7+401738 & 1.2 & 47.2 & 43.1 & I & 0.80 & $¡ 0.1$ & $2.8_{-0.4}^{+0.5}$ & 5.02 & 41.03 & $\mathrm{P}$ \\
\hline 587736752468394214 & 241.406998 & 32.936256 & 0.053 & CXOMP J160537.6+325610 & 7.7 & 18.7 & 19.9 & $\ldots$ & 2.30 & $¡ 0.2$ & $1.9_{-0.7}^{+0.8}$ & 10.33 & 40.53 & $\mathrm{~T}$ \\
\hline 587736752468394267 & 241.437714 & 32.872177 & 0.115 & CXOMP J160545.0+325219 & 6.0 & 30.3 & 20.1 & $\ldots$ & 2.31 & ¡0.1 & $2.1_{-0.5}^{+0.5}$ & 16.30 & 41.41 & no-class \\
\hline 587736781993935128 & 236.260956 & 36.156464 & 0.060 & CXOMP J154502.6+360923 & 3.2 & 9.8 & 18.8 & $\ldots$ & 1.65 & ¡0.1 & $3.3_{-1.0}^{+1.2}$ & 2.61 & 39.55 & no-class \\
\hline 587736781993935188 & 236.282913 & 36.146473 & 0.069 & CXOMP J154507.8+360847 & 3.5 & 12.5 & 18.8 & $\ldots$ & 1.65 & $¡ 0.1$ & $3.6_{-1.1}^{+1.3}$ & 2.91 & 39.54 & no-class \\
\hline 587736941445447871 & 216.464218 & 35.567928 & 0.186 & CXOMP J142551.4+353404 & 3.2 & 7.8 & 50.3 & $\ldots$ & 1.10 & $¡ 0.2$ & $3.5_{-1.6}^{+1.8}$ & 1.20 & 40.22 & S \\
\hline 588007004192637004 & 243.921677 & 47.186592 & 0.198 & CXOMP J161541.2+471111 & 0.5 & 121.6 & 3.4 & I & 1.23 & ¡0.1 & $1.7_{-0.3}^{+0.0}$ & 239.03 & 43.18 & $\mathrm{~T}$ \\
\hline 588007004192637199 & 243.922241 & 47.167870 & 0.197 & CXOMP J161541.3+471004 & 1.4 & 5.8 & 3.4 & $\ldots$ & 1.23 & ¡0.1 & $3.6_{-1.6}^{+3.9}$ & 6.97 & 40.99 & $\mathrm{P}$ \\
\hline 588007004694839559 & 119.126747 & 41.036259 & 0.072 & CXOMP J075630.4+410210 & 3.3 & 109.5 & 6.2 & $\ldots$ & 4.48 & ¡0.04 & $2.5_{-0.4}^{+0.5}$ & 102.97 & 41.65 & $\mathrm{~L}$ \\
\hline 588007005769957640 & 120.440094 & 44.110588 & 0.131 & CXOMP J080145.6+440638 & 6.6 & 11.8 & 9.2 & $\ldots$ & 4.73 & $¡ 0.1$ & $3.5_{-1.0}^{+1.2}$ & 7.35 & 40.67 & $\mathrm{P}$ \\
\hline 588010879292932251 & 173.027710 & 4.893930 & 0.150 & CXOMP J113206.6+045338 & 3.4 & 6.5 & 6.3 & $\ldots$ & 3.43 & $¡ 0.2$ & $3.2_{-1.4}^{+2.4}$ & 4.43 & 40.68 & no-class \\
\hline 588016891170914329 & 143.504242 & 33.990730 & 0.027 & CXOMP J093401.0+335926 & 5.9 & 13.6 & 31.8 & $\ldots$ & 1.47 & ¡0.1 & $3.4_{-1.0}^{+1.2}$ & 4.19 & 38.94 & no-class \\
\hline 588017111297622182 & 189.892838 & 47.537556 & 0.131 & CXOMP J123934.2+473215 & 9.1 & 82.4 & 4.5 & $\ldots$ & 1.13 & $2.6_{-0.6}^{+0.7}$ & $0.2_{-0.3}^{+0.2}$ & 434.23 & 43.15 & $\mathrm{~S}$ \\
\hline 588017111298932927 & 194.301773 & 47.330669 & 0.131 & CXOMP J125712.4+471950 & 2.3 & 31.3 & 49.8 & $\ldots$ & 1.15 & $\jmath^{-0.1}$ & $2.2_{-0.5}^{+0.6}$ & 3.13 & 40.78 & no-class \\
\hline 588017111833968667 & 187.945435 & 47.927235 & 0.030 & CXOMP J123146.9+475538 & 5.5 & 9.5 & 6.5 & $\ldots$ & 1.17 & $¡ 0.7$ & $-0.2_{-1.2}^{+0.9}$ & 34.97 & 40.81 & $\mathrm{~S}$ \\
\hline 588017567628591240 & 164.638321 & 12.720986 & 0.119 & CXOMP J105833.1+124315 & 6.2 & 8.8 & 4.9 & $\ldots$ & 2.13 & ¡0.5 & $1.9_{-0.9}^{+1.0}$ & 29.98 & 41.76 & $\mathrm{~T}$ \\
\hline
\end{tabular}


TABLE $1-$ Continued

\begin{tabular}{|c|c|c|c|c|c|c|c|c|c|c|c|c|c|c|}
\hline $\begin{array}{c}\text { ObjID } \\
(1)\end{array}$ & $\begin{array}{l}\mathrm{ra} \\
(2)\end{array}$ & $\begin{array}{l}\text { dec } \\
(3)\end{array}$ & $\begin{array}{c}z \\
(4)\end{array}$ & $\begin{array}{c}\text { srcid } \\
(5)\end{array}$ & $\begin{array}{c}\text { OAA } \\
(6)\end{array}$ & $\begin{array}{c}\text { counts } \\
(7)\end{array}$ & $\begin{array}{c}\text { Exp. } \\
(8)\end{array}$ & $\begin{array}{c}\text { Targ. } \\
(9)\end{array}$ & $\begin{array}{c}N_{H}^{G a l} \\
(10)\end{array}$ & $\begin{array}{c}N_{H}^{\text {Intr. }} \\
(11)\end{array}$ & $\begin{array}{c}\Gamma \\
(12)\end{array}$ & $\begin{array}{c}f_{x} \\
(13)\end{array}$ & $\begin{array}{l}L_{X} \\
(14)\end{array}$ & $\begin{array}{l}\text { Opt. Class } \\
\text { (15) }\end{array}$ \\
\hline 588017569779023967 & 201.370422 & 11.335633 & 0.086 & CXOMP J132528.9+112008 & 5.5 & 75.7 & 4.4 & $\ldots$ & 1.92 & $¡ 0.2$ & $1.9_{-0.3}^{+0.3}$ & 124.39 & 42.06 & S \\
\hline 588017604148133957 & 186.976318 & 44.363770 & 0.276 & CXOMP J122754.3+442149 & 1.7 & 21.6 & 4.8 & $\ldots$ & 1.34 & $¡ 1.3$ & $1.4_{-0.5}^{+0.5}$ & 48.44 & 42.83 & $\mathrm{~T}$ \\
\hline 588017605772837051 & 228.612808 & 36.634247 & 0.161 & CXOMP J151427.0+363803 & 2.5 & 21.0 & 43.5 & $\ldots$ & 1.35 & $¡ 0.2$ & $2.4_{-0.7}^{+0.8}$ & 2.28 & 40.80 & $\mathrm{P}$ \\
\hline 588017625613795424 & 170.110840 & 43.255371 & 0.145 & CXOMP J112026.6+431519 & 7.0 & 1.8 & 17.6 & $\ldots$ & 1.96 & ¡39.4 & $-0.7_{-3.0}^{+2.1}$ & 3.62 & 41.14 & $\mathrm{~S}$ \\
\hline 588017720102813842 & 168.741943 & 40.603119 & 0.075 & CXOMP J111458.0+403611 & 4.9 & 22.1 & 28.3 & $\ldots$ & 1.94 & $¡ 0.8$ & $0.7_{-0.5}^{+0.5}$ & 10.14 & 41.03 & S \\
\hline 588017722259865662 & 198.026779 & 42.690838 & 0.179 & CXOMP J131206.4+424127 & 3.2 & 15.4 & 80.6 & $\ldots$ & 1.37 & ¡2.2 & $-0.3_{-1.0}^{+0.7}$ & 4.76 & 41.44 & no-class \\
\hline 588017722259865691 & 198.164169 & 42.713638 & 0.111 & CXOMP J131239.3+424248 & 3.0 & 64.5 & 90.7 & $\ldots$ & 1.37 & $¡ 0.3$ & $1.6_{-0.3}^{+0.3}$ & 6.32 & 41.08 & $\mathrm{~L}$ \\
\hline 588017947748139147 & 205.227905 & 40.109875 & 0.170 & CXOMP J134054.6+400635 & 10.5 & 917.5 & 45.1 & $\ldots$ & 0.79 & $0.04_{-0.0}^{+0.0}$ & $2.0_{-0.1}^{+0.2}$ & 154.08 & 42.80 & $\mathrm{~L}$ \\
\hline 588018089466658843 & 231.747757 & 35.976982 & 0.055 & CXOMP J152659.4+355837 & 0.6 & 22.4 & 9.1 & I & 1.56 & $i^{-0.1}$ & $2.8_{-0.6}^{+0.1}$ & 11.71 & 40.35 & $\mathrm{~T}$ \\
\hline 588295840714129492 & 185.380676 & 49.176918 & 0.184 & CXOMP J122131.3+491036 & 4.4 & 17.2 & 72.2 & $\ldots$ & 1.42 & $¡ 0.1$ & $3.1_{-0.9}^{+1.0}$ & 1.97 & 40.60 & $\mathrm{~T}$ \\
\hline 588295840714129502 & 185.415253 & 49.332184 & 0.124 & CXOMP J122139.6+491955 & 5.0 & 15.3 & 77.0 & $\ldots$ & 1.44 & ¡3.1 & $0.5_{-0.8}^{+0.8}$ & 4.16 & 41.09 & $\mathrm{~T}$ \\
\hline 588295842853224515 & 156.433044 & 47.326378 & 0.062 & CXOMP J102543.9+471934 & 5.7 & 2.9 & 1.9 & $\ldots$ & 1.26 & ¡0.3 & $3.7_{-2.0}^{+2.6}$ & 12.03 & 40.00 & $\mathrm{P}$ \\
\hline 588297863638089749 & 126.979630 & 29.449829 & 0.029 & CXOMP J082755.1+292659 & 9.8 & 15.0 & 14.6 & $\ldots$ & 3.73 & $¡ 0.2$ & $2.3_{-0.9}^{+1.3}$ & 8.64 & 39.84 & $\mathrm{H}$ II \\
\hline 588848898846752926 & 197.902756 & -0.922150 & 0.083 & CXOMP J131136.6-005519 & 8.6 & 100.3 & 20.1 & $\ldots$ & 1.78 & $7.3_{-3.9}^{+5.2}$ & $0.2_{-0.7}^{+0.9}$ & 162.69 & 42.34 & $\mathrm{~S}$ \\
\hline 588848898846818469 & 198.065002 & -0.930759 & 0.081 & CXOMP J131215.6-005550 & 4.0 & 3.6 & 20.7 & $\ldots$ & 1.79 & $¡ 0.2$ & $4.2_{-1.8}^{+2.8}$ & 2.26 & 39.28 & $\mathrm{P}$ \\
\hline 588848899377529043 & 183.972015 & -0.601967 & 0.119 & CXOMP J121553.2-003607 & 3.0 & 8.1 & 42.0 & $\ldots$ & 2.08 & $0.6_{-0.6}^{+1.0}$ & $1.4_{-0.9}^{+0.9}$ & 2.38 & 40.75 & $\mathrm{~L}$ \\
\hline 588848899377529068 & 184.006836 & -0.625664 & 0.121 & CXOMP J121601.6-003732 & 4.9 & 17.4 & 41.6 & $\ldots$ & 2.09 & $i^{-0.0}$ & $4.9_{-1.0}^{+1.8}$ & 5.90 & 39.71 & $\mathrm{P}$ \\
\hline 588848899387228268 & 206.030533 & -0.475767 & 0.101 & CXOMP J134407.3-002832 & 0.5 & 3.6 & 8.7 & $\ldots$ & 2.02 & ¡2.5 & $1.2_{-1.2}^{+1.0}$ & 3.75 & 40.82 & $\mathrm{~T}$ \\
\hline 588848901519179802 & 170.660416 & 1.059925 & 0.075 & CXOMP J112238.4+010335 & 3.4 & 69.2 & 18.5 & $\ldots$ & 4.10 & $¡ 0.1$ & $1.8_{-0.3}^{+0.3}$ & 39.08 & 41.46 & $\mathrm{~T}$ \\
\hline 588848901519179967 & 170.649841 & 1.116308 & 0.039 & CXOMP J112235.9+010658 & 3.4 & 6.3 & 19.9 & $\ldots$ & 4.11 & ¡0.5 & $2.5_{-1.1}^{+1.3}$ & 3.05 & 39.58 & $\mathrm{H}$ II \\
\hline 588848901528289406 & 191.438202 & 1.079846 & 0.106 & CXOMP J124545.1+010447 & 1.1 & 9.7 & 6.3 & $\ldots$ & 1.69 & $¡ 0.1$ & $3.5_{-1.1}^{+1.1}$ & 6.31 & 40.35 & $\mathrm{~T}$ \\
\hline 588848901531107518 & 197.878723 & 1.184934 & 0.070 & CXOMP J131130.8+011105 & 8.5 & 24.7 & 4.5 & $\ldots$ & 1.97 & $15.1_{-5.8}^{+8.5}$ & $-1.5_{-0.8}^{+0.7}$ & 270.20 & 42.40 & $\mathrm{~S}$ \\
\hline
\end{tabular}

Note. - (1) SDSS Object ID. (2) and (3) J2000 epoch. (4) spectroscopic redshift. (5) ChaMP X-ray source ID, expressed as CXOMP Jhhmmss.s+/-ddmmss, using the truncated X-ray source position. (6) Chandra off-axis angle in arcmin; (7) net $0.5-8 \mathrm{keV}$ source counts. (8) Vignetting-corrected exposure time in ksec. (9) I = intended Chandra PI target. (10) Galactic column in units of $10^{20} \mathrm{~cm}^{-2}$. (11) Best-fit YAXX intrinsic column density in $10^{22} \mathrm{~cm}^{-2}$; upper limits are at $90 \%$ confidence level and errors represent 1- $\sigma$ uncertainties. (12) Best-fit YAXX power-law index $\Gamma$; errors represent $1-\sigma$ uncertainties. (13) X-ray flux $(0.5-8 \mathrm{keV})$ in units of $10^{-15} \mathrm{erg} \mathrm{s}^{-1} \mathrm{~cm}^{-2}$. (14) log X-ray luminosity (0.5 - $8 \mathrm{keV}$ ) in erg s${ }^{-1}$. (15) Optical spectral classification, $\mathrm{S}=$ Seyfert, $\mathrm{L}=$ LINER, $\mathrm{T}=$ Transition Object, $\mathrm{P}=$ Passive galaxy; see text for details. Note that only for objects with $i 200$ counts, $N_{H}^{I n t r}$. and $\Gamma$ values are the result of fitting simultaneously a power-law and absorption. 
TABLE 2

Object Sample Statistics.

\begin{tabular}{lccc}
\hline \hline \multicolumn{1}{c}{ Sample } & $N_{\text {opt }}\left(\right.$ fraction $\left.^{\mathrm{a}}, \%\right)$ & $N_{\mathrm{X}-\text { ray }}$ det. $\left(\right.$ fraction $\left.^{\mathrm{b}}, \%\right)$ & $N_{\mathrm{X}-\text { ray det. }} / N_{\text {opt }}(\%)$ \\
\hline H II & $498(27.6)$ & $7(6.5)$ & $1.4 \pm 4.5$ \\
Seyfert & $28(\mathbf{1 . 5})$ & $18(\mathbf{1 6 . 8})$ & $\mathbf{6 4 . 3} \pm \mathbf{1 8 . 9}$ \\
Transition & $194(10.7)$ & $32(29.9)$ & $16.5 \pm 7.2$ \\
LINER & $70(3.9)$ & $13(12.1)$ & $18.6 \pm 11.9$ \\
some emision-no class & $558(31.1)$ & $19(17.8)$ & $3.4 \pm 4.2$ \\
Passive & $459(25.4)$ & $18(16.8)$ & \\
& & & \\
\hline
\end{tabular}

Note. - The quoted errors represent standard deviations assuming Poisson statistics.

${ }^{\text {a }}$ Fraction by type of all 1807 SDSS galaxies on ACIS chips, which excludes those falling on $c c d=8$, and with $\theta>0.2$ deg.

b Fraction of all 107 X-ray detected SDSS galaxies.

TABLE 3

$\Gamma-L / L_{\text {edd }}$ - Correlation Coefficients and Significance

\begin{tabular}{lccc}
\hline \multicolumn{1}{c}{ Sample } & $r_{s}$ & Prob. & $\mathrm{N}$ \\
\hline for $L_{X}=L_{X}(0.5-8) \mathrm{keV} \mathrm{\ldots \ldots ...}$ \\
all & $\mathbf{- 0 . 5 2}$ & $<1.0 \times 10^{-3}$ & 107 \\
Seyfert & -0.18 & 0.43 & 18 \\
Transition & -0.29 & 0.11 & 31 \\
LINER & -0.39 & 0.18 & 13 \\
for $L_{X}=L_{X}(2-10) \mathrm{keV} \ldots \ldots \ldots$. \\
all & $\mathbf{- 0 . 7 5}$ & $<1.0 \times 10^{-3}$ & 107 \\
Seyfert & $\mathbf{- 0 . 3 6}$ & 0.12 & 18 \\
Transition & $\mathbf{- 0 . 5 2}$ & $2.3 \times 10^{-3}$ & 31 \\
LINER & $\mathbf{- 0 . 5 6}$ & $4.4 \times 10^{-2}$ & 13 \\
& & & \\
\hline
\end{tabular}

Note. - The Spearman-rank test correlation coefficient, chance probability, and number of sources for each correlation/sample respectively. The most significant probabilities for anticorrelations (chance probability less than $5 \%$ ) are shown in boldface.

TABLE 4

$\Gamma-L / L_{\text {edd }}$ - Linear Regression Coefficients

\begin{tabular}{lccc}
\hline \hline \multicolumn{1}{c}{ Sample } & Slope & Intercept & $\chi^{2} /$ dof \\
\hline for $L_{X}=L_{X}(0.5-8) \mathrm{keV}$ & $\ldots \ldots .$. & & \\
all & $-0.16 \pm 0.04$ & $1.36 \pm 0.13$ & $309 / 105$ \\
all, no passive, no HII & $-0.07 \pm 0.05$ & $1.51 \pm 0.15$ & $235 / 81$ \\
all with $L_{X} \gtrsim 10^{42} \mathrm{erg} \mathrm{s}^{-1}$ & $0.12 \pm 0.12$ & $2.09 \pm 0.29$ & $83 / 17$ \\
Seyfert & $0.94 \pm 0.15$ & $3.59 \pm 0.35$ & $89 / 16$ \\
Transition & $-0.001 \pm 0.11$ & $1.88 \pm 0.37$ & $21 / 29$ \\
LINER & $-0.20 \pm 0.09$ & $1.42 \pm 0.34$ & $29 / 11$ \\
for $L_{X}=L_{X}(2-10) \mathrm{keV}$ & $\ldots \ldots \ldots .$. & & \\
all & $-0.27 \pm 0.04$ & $0.98 \pm 0.13$ & $274 / 105$ \\
all, no passive, no HiI & $-0.21 \pm 0.05$ & $1.11 \pm 0.15$ & $219 / 81$ \\
all with $L_{X} \gtrsim 10^{42} \mathrm{erg} \mathrm{s}^{-1}$ & $-0.19 \pm 0.14$ & $1.28 \pm 0.36$ & $95 / 16$ \\
Seyfert & $0.42 \pm 0.18$ & $2.49 \pm 0.43$ & $119 / 16$ \\
Transition & $-0.09 \pm 0.10$ & $1.57 \pm 0.38$ & $21 / 29$ \\
LINER & $-0.25 \pm 0.09$ & $1.18 \pm 0.34$ & $26 / 11$ \\
& & & \\
\hline
\end{tabular}

Note. - The slope, intercept, $\chi^{s}$, and degrees of freedom (dof) for the best error-weighted linear fits for the whole sample of X-ray detected galaxies, and per spectral type. Errors are at 1- $\sigma$ confidence levels. 


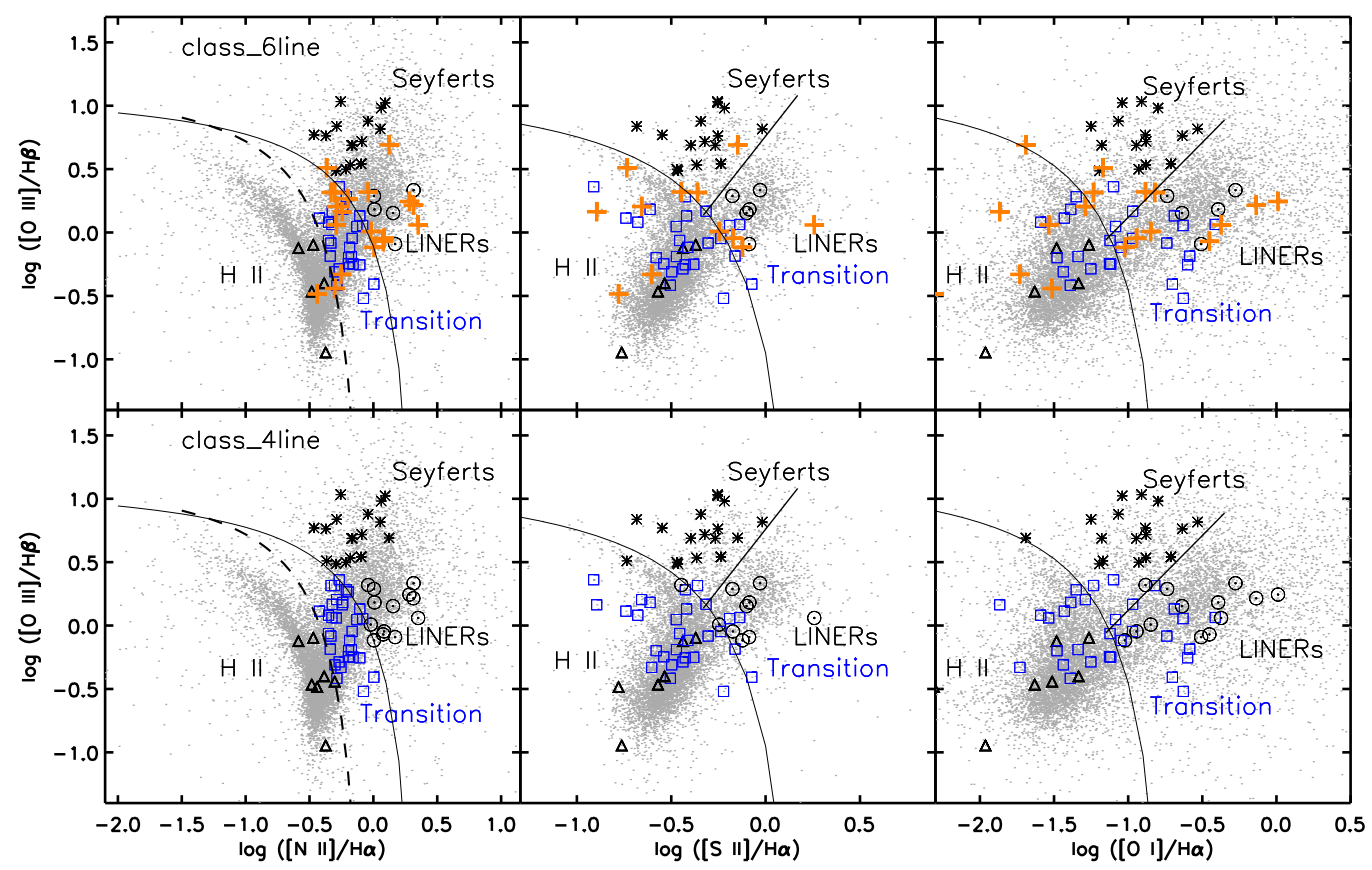

FIG. 1.- Diagnostic diagrams for all ChaMP galaxies that exhibit emission-line activity, with relatively high $(>2)$ signal-to-noise line flux measurements in all 6 lines $(t o p)$, and in only [N II], [O III], $\mathrm{H} \beta$, and $\mathrm{H} \alpha$ (bottom). The solid and dashed black curves illustrate the Kewlev et al. (2001) and Kauffmann et al. (2003b) separation lines while the diagonal lines illustrate the separation between Seyferts and LINERs by Kewlev et al. (2006). The grey points correspond to galaxies in ChaMP fields that fall on or near the ChaMP ACIS chips, but are not necessarily X-ray detected. Seyferts, Liners, Transition Objects, and H II galaxies are shown as asterixes, open circles, blue squares, and triangles, respectively. The orange crosses are 6-line non-classified objects with a 4-line class. It is quite apparent that the combination of the 4-line classification and X-ray detection is very efficient in distinguishing between different types of emission among LLAGN, quantitatively identical to the 6-line method. 

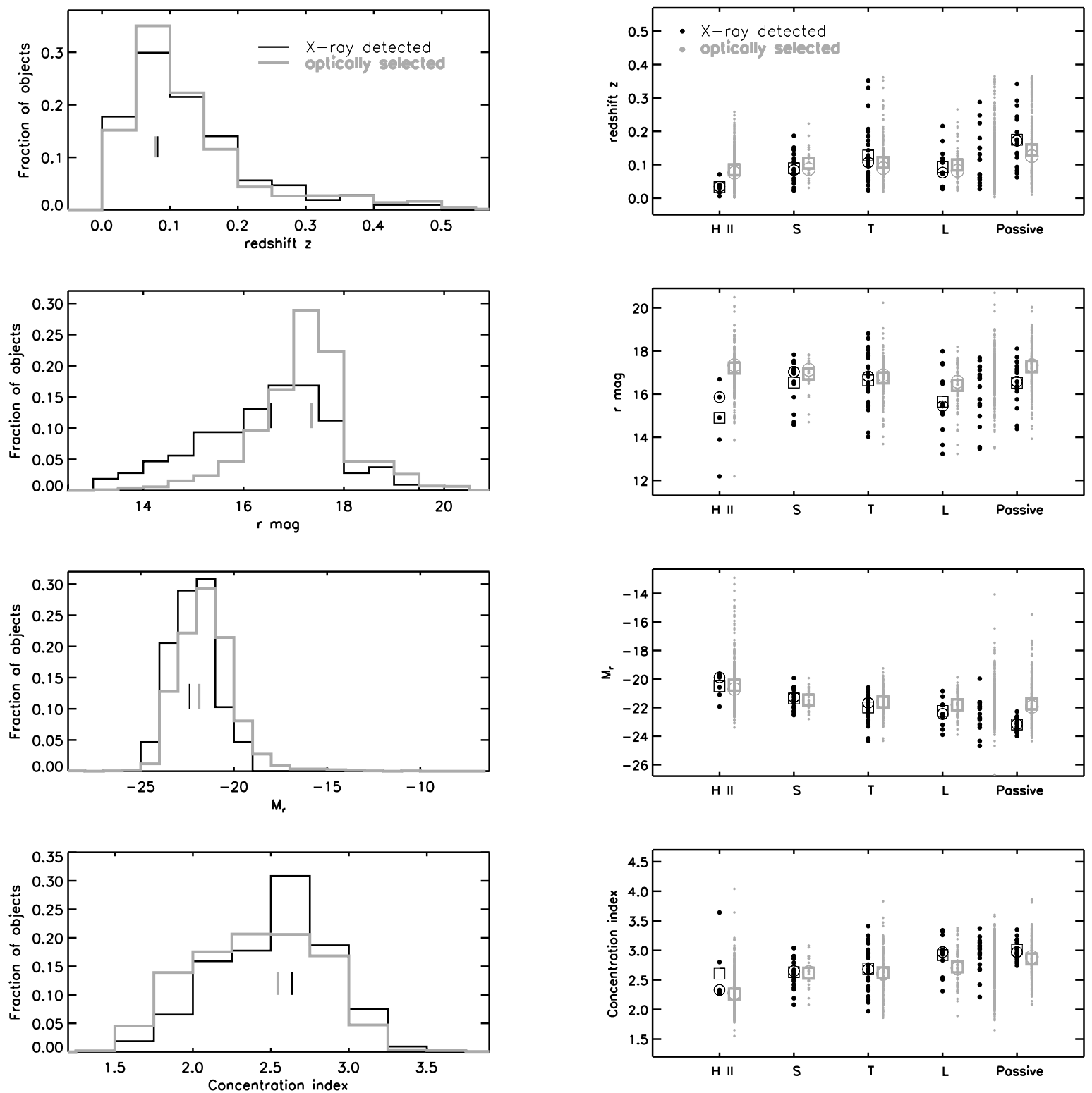

FIG. 2.- Comparison of the optical properties of X-ray detected galaxies and all optically selected (SDSS) galaxies on or near ChaMP fields/chips. Left: Histograms of redshift $(z)$, apparent $r$-band magnitude $\left(m_{r}\right)$, absolute $r$-band magnitude $\left(M_{r}\right)$, and the concentration index $(C)$ for the full samples of SDSS galaxies on/near ChaMP fields and the ChaMP detections; median values are indicated by the vertical bars. Right: Individual measurements of $z, m_{r}, M_{r}$ and $C$ are shown separately per galaxy spectral type; both average (squares) and median (circles) values are indicated here, for all types of objects. The bias caused by the X-ray detection criterion is clearly weak; the few X-ray detected HıI's dominate the observed difference in $r$ and $M_{r}$ distributions. 

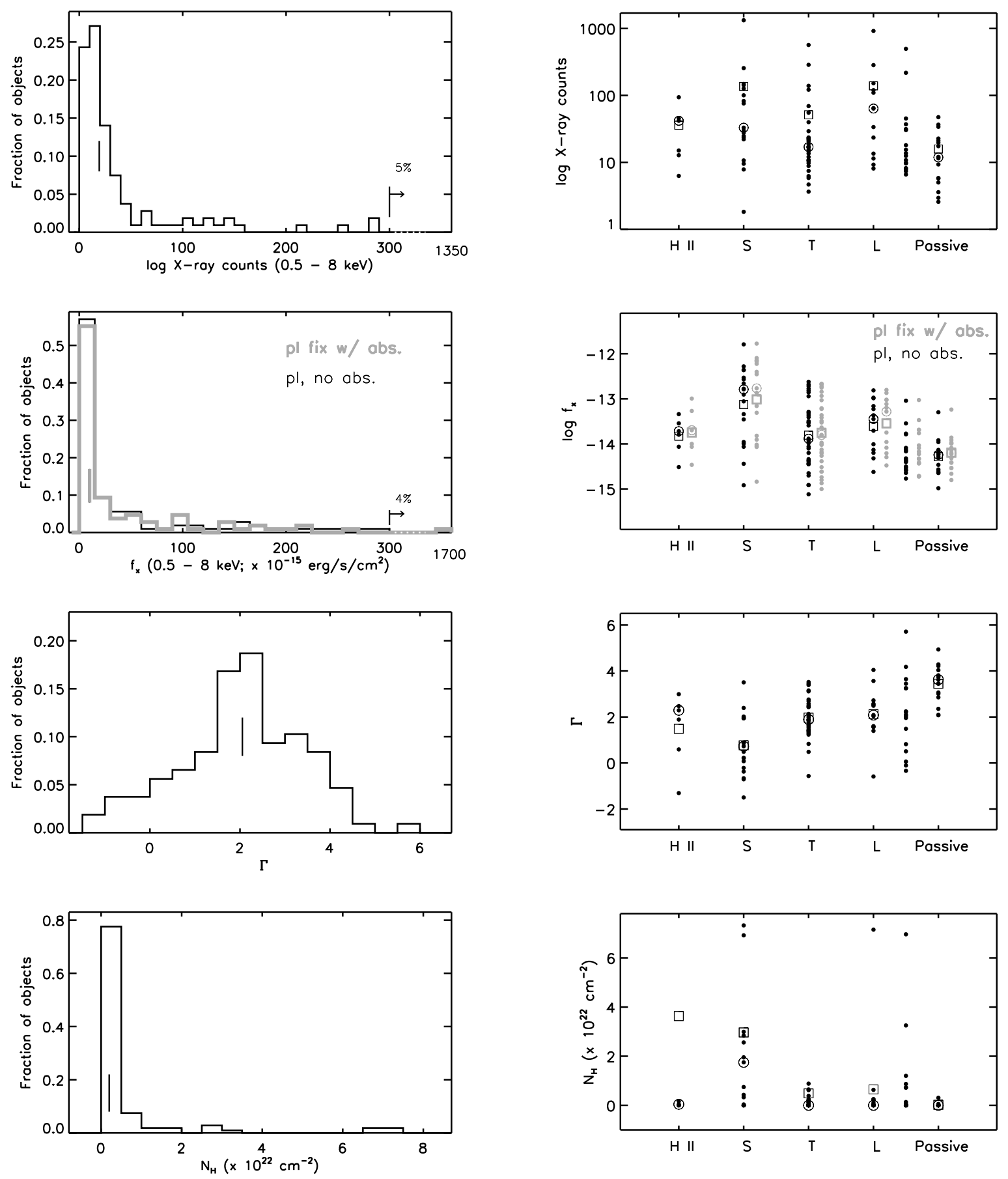

Fig. 3.- Distribution of X-ray properties of the ChaMP galaxies. Left: Histograms of X-ray counts in the $0.5-8 \mathrm{keV}$ regime, the $\mathrm{X}$-ray flux $f_{x}$ (calculated via two fitting models, a power-law with no intrinsic absorption and a fixed power-law with variable absorption), the (best) X-ray photon index $\Gamma$, and the (best) $N_{H}$ (see text); median values are indicated by the vertical bars. Right: Individual measurements of X-ray counts, $f_{x}, \Gamma$, and $N_{H}$ shown separately per galaxy spectral type; average and median values are indicated for all types of objects by squares and circles respectively. 

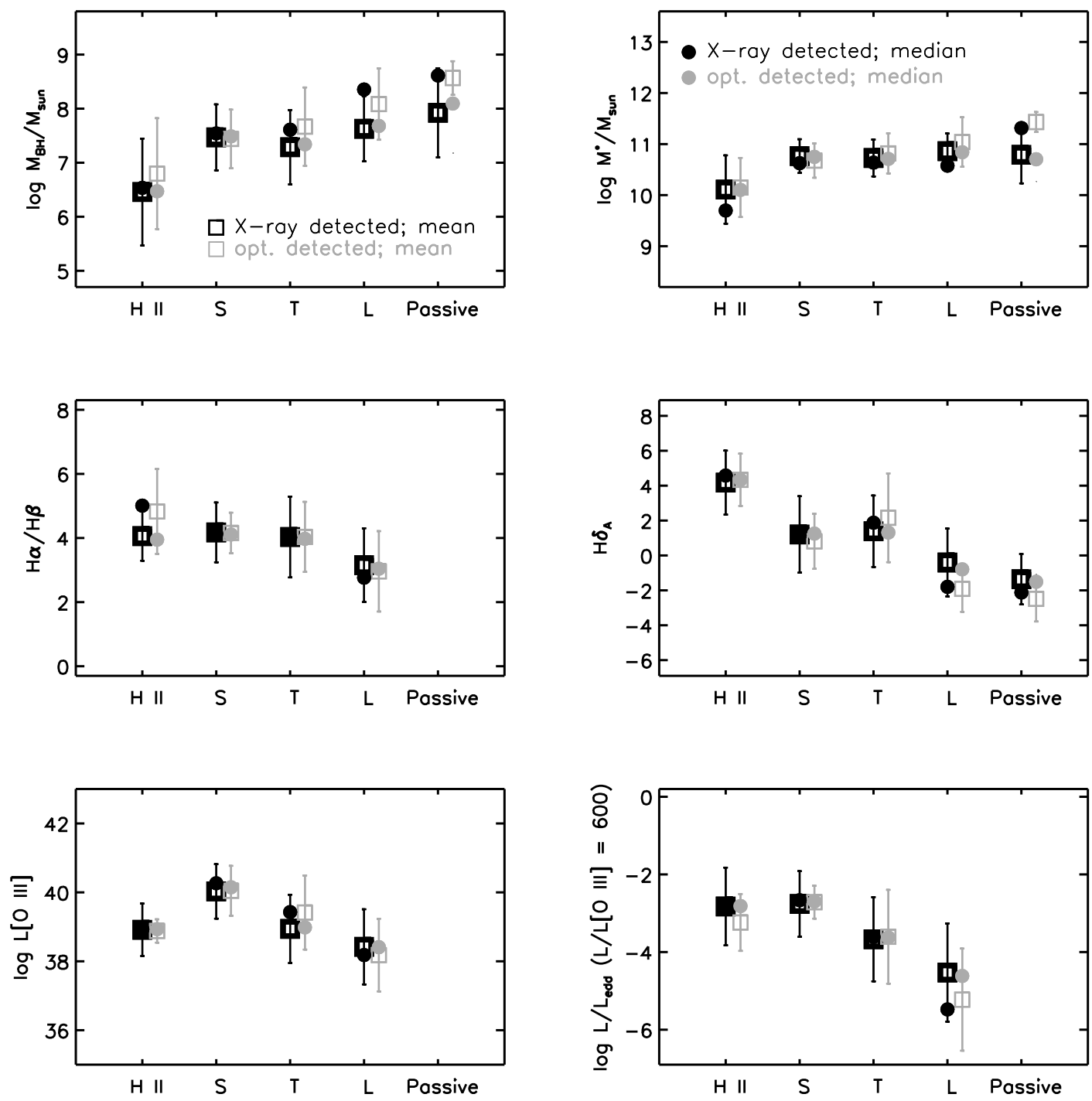

FIG. 4. - Comparison of median and average optical properties of X-ray detected galaxies and all optically selected galaxies on or near ChaMP fields/chips, along the $H$ II $\rightarrow S \rightarrow T \rightarrow L \rightarrow$ passive galaxies sequence. The plots show: the $\mathrm{BH}$ mass (based on $M_{\mathrm{BH}}-\sigma_{*}$ relation of Tremaine et al. 2002), the (dust corrected) stellar mass $\left(\log M_{*} / M_{\odot}\right)$, the Balmer decrement, the $\mathrm{H} \delta_{A}$ Balmer absorption-line index, $L[\mathrm{OIII}]$, and the accretion rate expressed by $L_{\mathrm{bol}} / L_{\mathrm{Edd}}$, where $L_{\mathrm{bol}} / L[O I I I]=600$; average and median values are indicated by squares and circles respectively. 


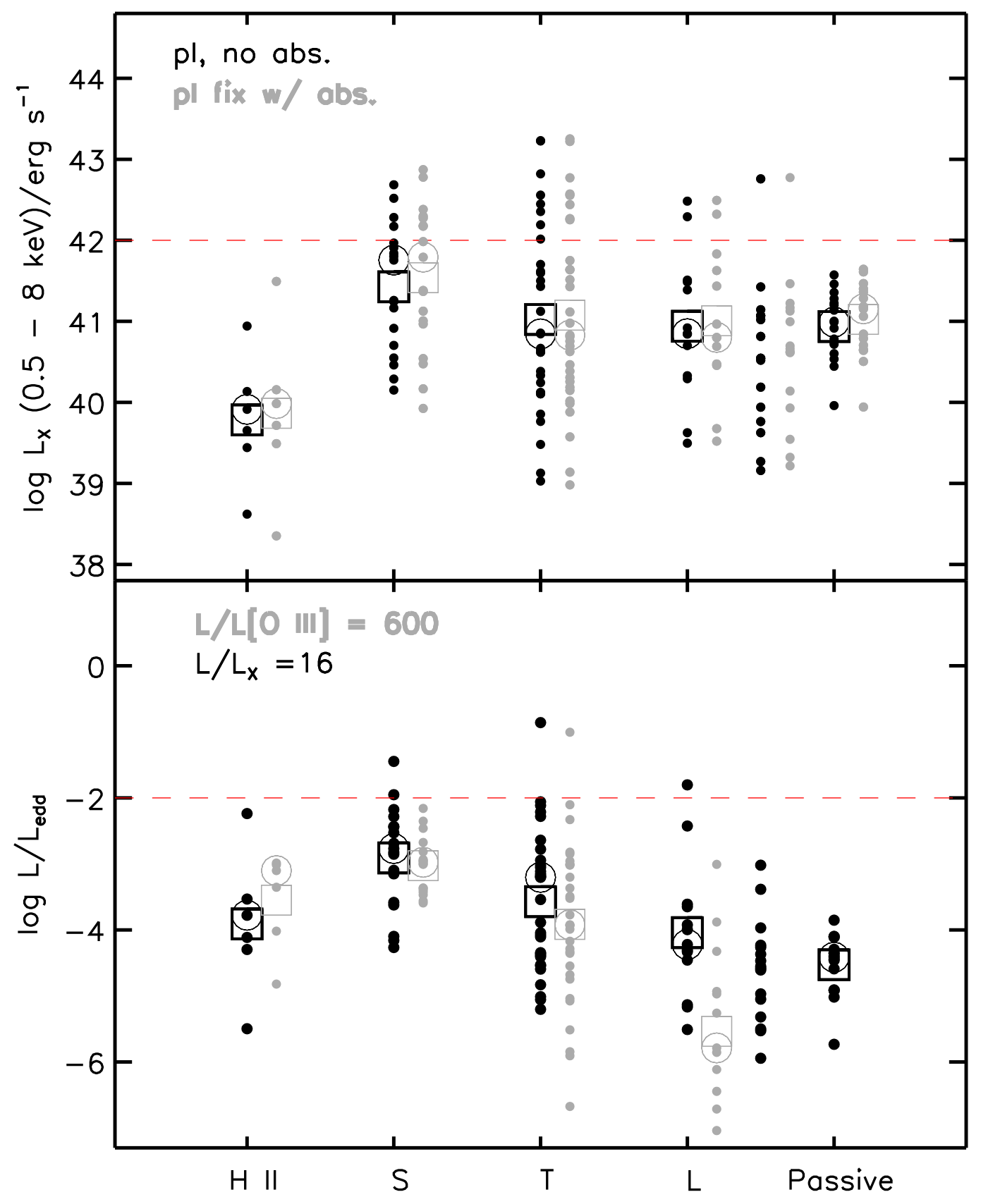

FIG. 5.- The $H$ II $\rightarrow S \rightarrow T \rightarrow L \rightarrow$ passive galaxies sequence in X-ray: the X-ray luminosities obtained via yaxx for two fitting models (1. a power-law with no intrinsic absorption and 2. a fixed power-law with $\Gamma=1.9$ with intrinsic absorption); the accretion rate values, expressed by $L / L_{\mathrm{Edd}}$, calculated using both $L_{\mathrm{bol}} / L_{X}=16$ and $L_{\mathrm{bol}} / L[O I I I]=600$ are compared; average and median values are indicated by squares and circles respectively. 


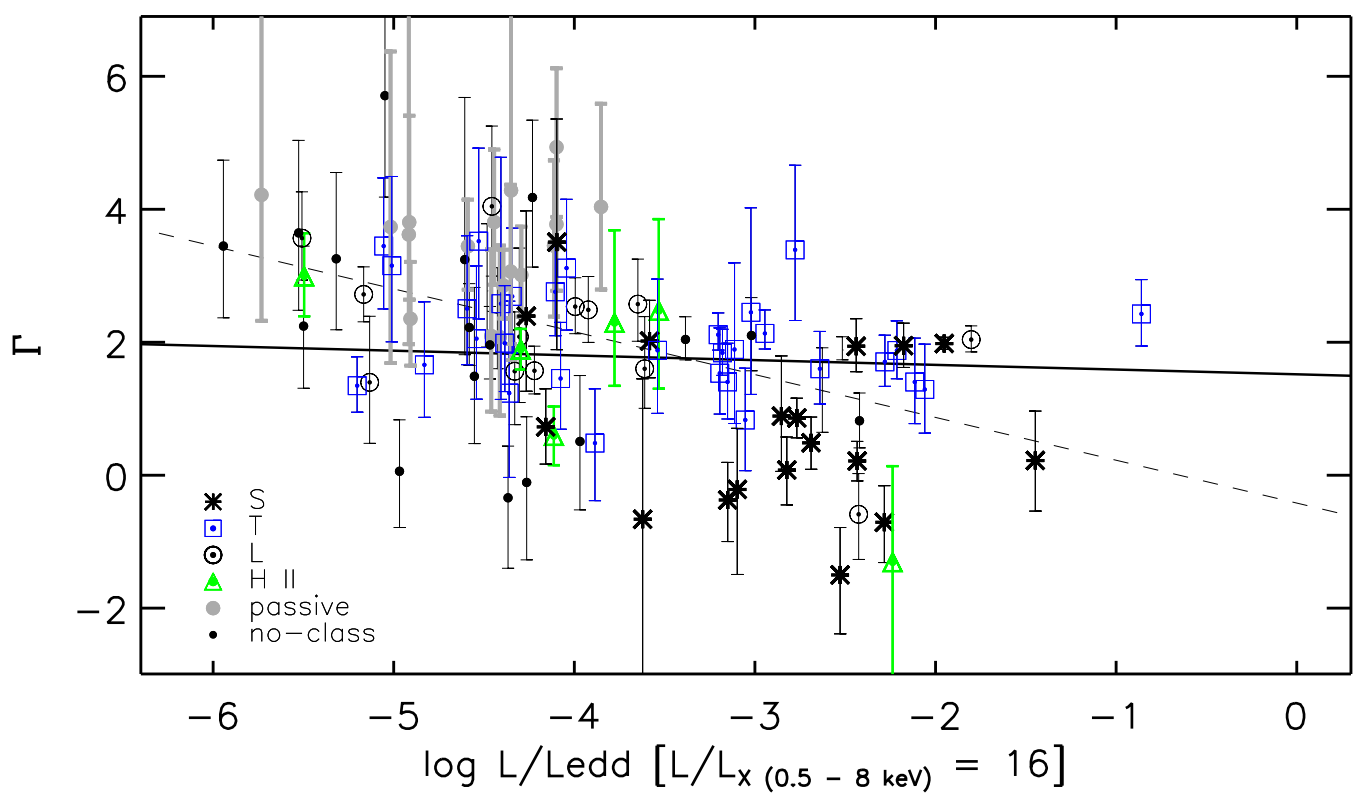

FIG. 6.- Distribution of the X-ray photon index as a function of $L / L_{\text {edd }}$ where $L=L_{\mathrm{bol}}$ is estimated using $L_{\mathrm{bol}} / L_{X}=16$. There is clearly apparent an anti-correlation between these two measures; the symbols reflect the different spectral optical classification. The solid and dotted lines reflect the best fit linear relations with the errors weighted and not weighted, respectively.

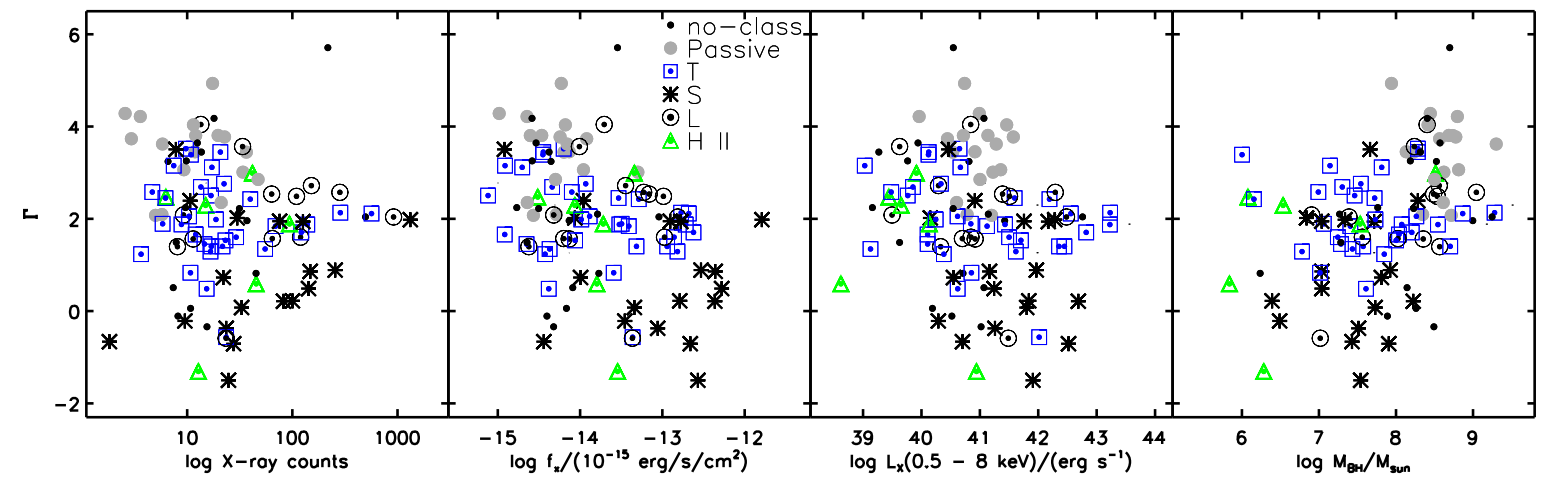

FIG. 7.- The relation between the $0.5-8 \mathrm{keV}$ X-ray photon index and the total number of counts, the $0.5-8 \mathrm{keV}$ flux $f_{x}$, the $0.5-8$ $\mathrm{keV}$ luminosity $L_{X}$, and the BH mass. No relation appears to exist between $\Gamma$ and X-ray counts, while there are rather weak apparent negative correlations with $f_{x}$ and $L_{X} ; M_{\mathrm{BH}}$ does not appear to correlate with $\Gamma$. 


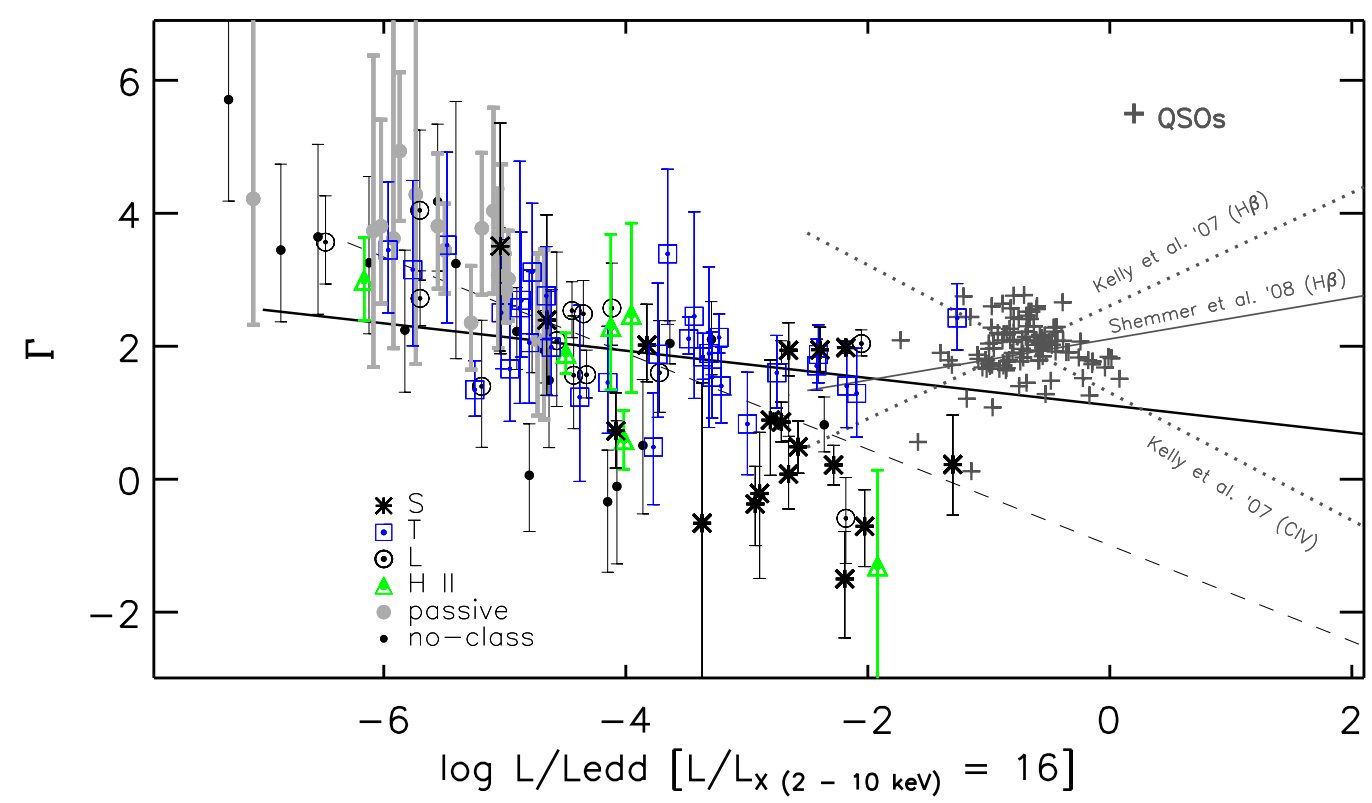

FIG. 8. - Distribution of the X-ray photon index $\Gamma$ as a function of $L / L_{\text {edd }}$ where $L=L_{\mathrm{bol}}$ is estimated using $L_{\mathrm{bol}} / L_{X}=16$, with $L_{X}=L_{X}(2-10 \mathrm{keV})$ calculated from $L_{X}(0.5-8 \mathrm{keV})$ using $\Gamma$. We show for comparison the measurements of $\Gamma$ and $L / L_{\mathrm{edd}}$ for the ChaMP (high $z$ ) SDSS QSOs with spectra (Green et al. 2009), where both $\Gamma$ and $L / L$ edd are calculated in the same way as for the ChaMP galaxies/LLAGN, with the difference that the bolometric correction for quasars is considered to be $L_{\mathrm{bol}} / L_{X}=83$, independend of their luminosity. 